Check for updates

Cite this: RSC Adv., 2017, 7, 48876

Received 17th September 2017 Accepted 11th October 2017

DOI: 10.1039/c7ra10315h

rsc.li/rsc-advances

\section{Effect of nanosilica content on the corrosion inhibition of composite coatings of a filled epoxy resin grafted with a hydrophobic fluoroalkylsilane: a dual critical concentrations interpretation}

\author{
Fuguo Deng, ${ }^{\text {abc }}$ Lishi Wang, ${ }^{c}$ Yong Zhou, ${ }^{d}$ Xinghou Gong, ${ }^{\text {abc }}$ Xipo Zhao, ${ }^{\text {abc }}$ Tao Hu ${ }^{\star a b c}$ \\ and Chonggang Wu iD *abc
}

3,3,3-Trifluoropropylmethyldimethoxysilane (TMDMS) was successfully grafted to molecules of an epoxy resin (EPR) of bisphenol-A origin with an epoxy value of 0.440 , i.e. EP44, by transesterification (i.e. alcoholysis) of the TMDMS methoxyls with the EP44 hydroxyls under anhydrous dibutyltin-dilaurate catalysis. Meanwhile, a nanosilica powder was modified with 1,1,1,3,3,3-hexamethyldisilazane (HMDS) via electrophilic substitution of the nanosilica hydroxyls by the HMDS trimethylsilyls to significantly decrease its surface hydroxyls for controlled aggregation. Six coatings, onto an $\mathrm{NaOH}$ solution-treated tinplate substrate, of EP44, of the TMDMS-grafted EP44, and of the composites of the TMDMS-grafted EP44 filled with $0.5,1,3$ and 5 wt\% of the HMDS-modified nanosilica were then step-cured mildly with an amino-terminated polyamide from a solution. Owing to the introduction of superhydrophobic trifluoropropyls possibly plus a reduction in the hydrophilic hydroxyl concentration present, the hydrophobicity, evaluated by the water contact-angle, of the EP44 coating increased considerably upon its TMDMS grafting, which then changed little with further addition of the nanosilica. Electrochemical impedance spectroscopy data and simulations revealed that the anticorrosive performance of the TMDMS-grafted EP44 coating, upon immersion into an $\mathrm{NaCl}$ solution, was significantly improved compared with the EP44 coating, primarily due to its remarkably enhanced hydrophobic barrier to the water-mediated corrosives (water, $\mathrm{NaCl}$, oxygen, other molecules and ions, etc.). However, the anticorrosion behaviour of the composite coatings was dominated by a corrosion inhibition (i.e. physical barrier) mechanism by the nanosilica filling the pores (free volumes, voids, cracks, etc.) susceptible to the corrosives. As the nanosilica content steadily was raised from 0 to $5 \mathrm{wt} \%$, the corrosion inhibition of the composite coatings first intensified, probably thanks to an enhancement of the filling rate of the pores, and subsequently weakened, presumably due to an increase in the porosity from increased size exclusion of aggregated nanoparticles, and finally improved again possibly owing to a densification of the outside-of-pore barriers of greatly aggregated, size excluded particles, which constituted dual critical concentrations (DCCS) of the nanosilica at 0-0.5 and $\sim 3 \mathrm{wt} \%$, respectively, that gave rise to a maximum followed by a minimum in the corrosion inhibition. To our knowledge, this has been the first work reporting this unique DCCs behaviour for EPR/nanosilica coatings, which, dictated by a hydrophobic EPR matrix of small porosity as well as a modified nanosilica of controlled aggregation, may universally be extended to the corrosion inhibition of other polymer/nanoceramic coatings.
${ }^{a}$ Hubei Provincial Key Laboratory of Green Materials for Light Industry, Hubei University of Technology, Wuhan, Hubei 430068, China. E-mail: hutao@mail.hbut. edu.cn; cgwu@mail.hbut.edu.cn

${ }^{b}$ Collaborative Innovation Center of Green Light-weight Materials and Processing, Hubei University of Technology, Wuhan, Hubei 430068, China

${ }^{c}$ School of Materials and Chemical Engineering, Hubei University of Technology, Wuhan, Hubei 430068, China

${ }^{d}$ Key Laboratory for Green Chemical Process of Ministry of Education, Wuhan Institute of Technology, Wuhan, Hubei 430205, China

\section{Introduction}

Metals have found extensive applications in construction, microelectronics, aerospace, automotives, etc. industries. ${ }^{1-3}$ However, owing to their relatively high chemical activity, metals are subject to corrosion, which has resulted in their increased consumption causing great economic losses and may even lead to catastrophic accidents. ${ }^{4}$ The first practical and widely used method to inhibit metal corrosion was based on self-healing chromate conversion coatings, which, nevertheless, have been 
restricted or entirely banned in many countries due to the presence of carcinogenic and environmentally detrimental hexavalent chromium ions $\left(\mathrm{Cr}^{6+}\right) .^{2,5-11}$ Great efforts have been made to seek alternative candidates of promising and durable corrosion protection including organic coatings, more friendly inorganic conversion coatings, organic-inorganic hybrid coatings, conducting polymer coatings, etc.

Organic coatings ${ }^{\mathbf{1 2 , 1 3}}$ have increasingly become an effective solution for corrosion protection, of which the coatings of epoxy resin (EPR) are the most popular instance applied due to its excellent chemical resistance, strong adhesion to metallic substrates, low curing shrinkage, etc. ${ }^{14}$ However, due to its rich presence of hydrophilic hydroxyl groups, ${ }^{14,15}$ a cured EPR displays such poor ageing ${ }^{16}$ and heat ${ }^{17}$ resistance as well as high brittleness that its coatings are difficult to produce long lasting corrosion protection. To overcome this, silicone resin (SR), i.e. polysiloxane, by virtue of its superior weather resistance, high thermal stability, good toughness, and strong hydrophobicity, has most typically been incorporated to improve the anticorrosive performance of EPR coatings by application of an SR primer underneath an EPR finish, ${ }^{\mathbf{8 , 1 8}}$ by blending as a dispersed gel phase with an EPR, ${ }^{9,19}$ by formation of an interpenetrating polymer network with an $\mathrm{EPR},{ }^{20}$ by (block or graft) copolymerisation with an EPR during its cure, ${ }^{21,22}$ etc. In the last decade, organosilane small molecules, ${ }^{\mathbf{1 4 , 2 3}}$ e.g. those of (3-glycidoxypropyl)trimethoxysilane (GPTMS), ${ }^{\mathbf{1 4}}$ have interestingly been grafted onto EPR molecules prior to their cure via transesterification (i.e. alcoholysis) reaction of the alkoxy (e.g. methoxy) groups of the former with the hydroxyls of the latter anhydrously catalysed by dibutyltin dilaurate (DBTDL); accordingly, Ji et al. ${ }^{\mathbf{1 4 , 2 3}}$ claimed that the absorption of water, medium for corrosives, into the cured EPRs was decreased as a result of a consumption of their preexisting hydrophilic hydroxyls by the reaction, which favoured the anticorrosion of the EPR coatings. Nevertheless, this effect of the pre-cure decrease in the hydroxyl concentration presumably was insignificant since more than $80 \mathrm{~mol} \%$ of the hydroxyls present in the EPRs, which were obtained from graft modification with various organosilanes of the bisphenol-A based EPR of an epoxy value of 0.440 (i.e. EP44), were newly formed upon their cure. The above organosilane-grafted EPRs approach, ${ }^{14,23}$ although reported by a single research group, has provided a promising strategy for the chemical modification of the EPRs molecular structures.

Alternatively, inorganic ceramic (IC) nanofillers, at no more than $10 \mathrm{wt} \%$ loading levels, have been added to EPR matrices to form composite coatings for the corrosion inhibition of metallic substrates; this generally is because the nanosized particles, at their low concentrations and thus small sizes, effectively fill the as many as possible pores (free volumes, voids, cracks, etc.) of the EPRs, which leads to the significantly detoured diffusion channels, present in the composite coatings, that conduce to improved corrosion prevention of the metallic substrates. ${ }^{24-28} \mathrm{It}$ has further been found that, as the content of IC nanoparticles is increased steadily, the corrosion inhibition of EPR/IC composite coatings first improves and then deteriorates, exhibiting the maximum at an optimum (i.e. critical) IC concentration beyond which the porosity (from the failure of aggregated IC nanoparticles to fill the smaller pores) of the composite coatings begins to increase gradually. ${ }^{\mathbf{2 4 2 9}}$ For instance, Ghanbari and Attar ${ }^{30}$ reported that the composite coatings of an EPR and a nanosilica powder treated with GPTMS demonstrated the maximum corrosion resistance at a critical nanosilica concentration of $\sim 4 \mathrm{wt} \%$.

In this work, we have accomplished successfully a novel modification of EPRs for anticorrosive coatings, which is a combination of the grafting of fluoroalkyl-containing organosilane onto and the incorporation of surface-treated IC nanofiller into EP44 molecules. Specifically, a 3,3,3-trifluoropropylmethyldimethoxysilane (TMDMS) grafted EP44 is studied against EP44 for improved corrosion protection, based on its hydrophilic hydroxyl concentration decrease from the graft reaction and the hydrophobic trifluoropropyl introduction jointly for hydrophobic barrier anticorrosion, as well as on its water consumption from hydrolysis of the residual TMDMS methoxy groups for water-digestive barrier anticorrosion. Further, the TMDMS-grafted EP44 is filled with different contents of 1,1,1,3,3,3-hexamethyldisilazane (HMDS) treated nanosilica for maximisation of the corrosion inhibition of the composite coatings.

\section{Experimental}

\section{Materials}

A bisphenol-A based EPR of an epoxy value of 0.440 (component $\mathrm{A}$ ), i.e. EP44, and its curing agent (component B), an aminoterminated polyamide-based compound (i.e. G650), were supplied by Nanjing Haozhuo Materials Technology Co., Ltd., China. 3,3,3-Trifluoropropylmethyldimethoxysilane (TMDMS) ( $\geq 99 \%$ ), dibutyltin dilaurate (DBTDL) (95\%), and 1,1,1,3,3,3hexamethyldisilazane (HMDS) (nitrogen flushed, 98\%), respectively, were obtained from Zhejiang Research Institute of Chemical Industry, China, Sigma-Aldrich, and Acros Organics. A nanosilica powder of $30 \mathrm{~nm}$ in mean diameter $(99.5 \%$ metals basis), potassium bromide ( $\mathrm{KBr})$ (specpure reagent), and deuterated chloroform (99.8\%) were purchased from Aladdin Industrial Corp., China. Tinplate $\left(120 \times 50 \times 2.8 \mathrm{~mm}^{3}\right)$ and sand papers of increasing meshes (in the order of $\# 240$, \#600, and \#1000) were supplied by Guangzhou DMY Instruments Co., Ltd., China and Hubei Yuli Abrasive Belts Group, China, respectively. Poly(vinyl chloride) (PVC) tubes of $40 \mathrm{~mm}$ in outer diameter and $36.6 \mathrm{~mm}$ in inner diameter, obtained from Yingda Plastic Building Materials Co., Ltd., China, were cut into $50 \mathrm{~mm}$ lengths. Sodium hydroxide $(\mathrm{NaOH})$ (analytical reagent, $\geq 96 \%$ ), sodium chloride $(\mathrm{NaCl})$ (analytical reagent, $\geq 99.5 \%$ ), potassium chloride $(\mathrm{KCl})$ (analytical reagent, $\geq 99.5 \%$ ), toluene (analytical reagent, $\geq 99.5 \%$ ), acetone (analytical reagent, $\geq 99.5 \%$ ), $n$-butanol (analytical reagent, $\geq 99.5 \%$ ), xylene (analytical reagent, $\geq 99 \%$ ), and molecular sieves (Type $4 \mathrm{~A}$ ) all were purchased from Sinopharm Chemical Reagents Co., Ltd., China. Distilled water was home made in our laboratory using a stainless steel water distiller. All the chemicals were used as received without further purification. 
Pre-cure grafting of 3,3,3-

trifluoropropylmethyldimethoxysilane onto epoxy resin molecules

A modified EPR was synthesised by reaction of an EPR with a fluorosilane monomer under anhydrous, DBTDL catalyst conditions, for which a process of chemical grafting of TMDMS to EP44 molecules is illustrated in Scheme 1a and the proposed chemical reaction in Scheme 1b. A solution of $61.0 \mathrm{~g}$ of EP44, $27.2 \mathrm{~g}$ of TMDMS, and $1.6 \mathrm{~g}$ of DBTDL in $60.4 \mathrm{~g}$ of toluene fully dried by Molecular sieves $4 \mathrm{~A}$ was added to a $250 \mathrm{~mL}$ threenecked flask equipped with a magnetic stirrer hotplate as well as a condenser. The mixture was stirred and refluxed at $95{ }^{\circ} \mathrm{C}$ (oil bath) for $8 \mathrm{~h}$, which was then rotary-evaporated, starting at $30{ }^{\circ} \mathrm{C}$ slowly heated to $75{ }^{\circ} \mathrm{C}$ within $\sim 1.5 \mathrm{~h}$, subsequently transferred to a beaker, and finally dried in vacuo at $50{ }^{\circ} \mathrm{C}$ overnight followed by at $80{ }^{\circ} \mathrm{C}$ for at least $48 \mathrm{~h}$, to thoroughly remove toluene, unreacted TMDMS, any by-product(s), etc. A colourless, viscous liquid product was thus obtained, which supposedly was a TMDMS-grafted EP44.

\section{Surface modification of a nanosilica powder with 1,1,1,3,3,3- hexamethyldisilazane}

A schematic of the surface modification process of the nanosilica powder with HMDS is shown in Scheme 2a, and the supposed reaction mechanism in Scheme $2 \mathrm{~b} .5 .2 \mathrm{~g}$ of the white nanosilica powder was vacuum-dried at $150{ }^{\circ} \mathrm{C}$ for $3 \mathrm{~h}$, which was then transferred, along with $35.8 \mathrm{~g}$ of HMDS, to a $250 \mathrm{~mL}$ three-necked flask equipped with a hotplate and a condenser. The resulting suspension was vigorously stirred mechanically

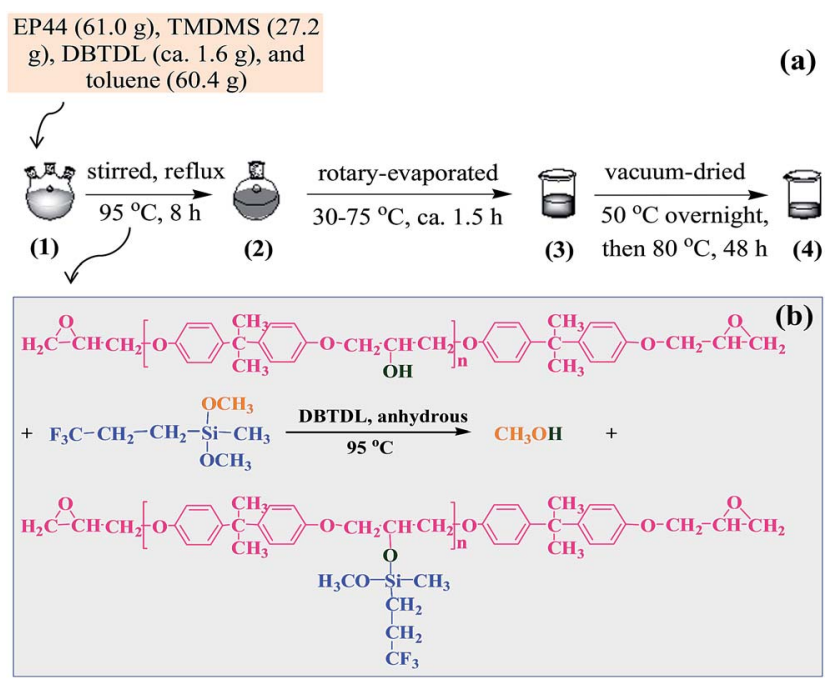

Scheme 1 (a) Illustration of a grafting process of 3,3,3-trifluoropropylmethyldimethoxysilane (TMDMS) onto the molecules of a bisphenol-A based epoxy resin of an epoxy value of 0.440 (i.e. EP44) prior to its cure, in toluene solution under anhydrous catalysis by dibutyltin dilaurate (DBTDL); (b) schematic of the graft reaction of TMDMS onto EP44 molecules (i.e. alcoholysis of TMDMS by EP44) to form a fluoroalkyl-containing EP44 of a significantly decreased hydroxyl concentration, which occurs during the process given in (a) from step 1 to 2 .

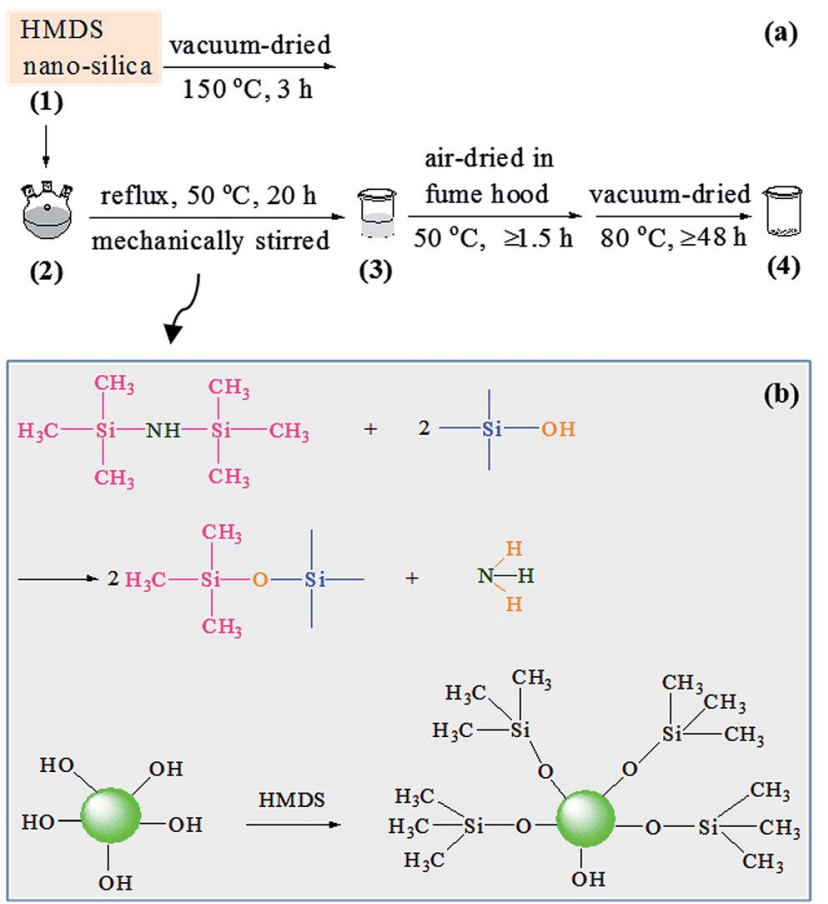

Scheme 2 (a) Illustration of the surface modification process of a nanosilica powder with 1,1,1,3,3,3-hexamethyldisilazane (HMDS); (b) schematic of the reaction of (the imino groups of) HMDS with (the hydroxy groups of) the nanosilica to modify the polar hydroxyls into non-polar methyls on the nanosilica powder surfaces, which occurs during the process given in (a) from step 2 to 3 .

and refluxed at $50{ }^{\circ} \mathrm{C}$ (oil bath) for $20 \mathrm{~h}$, which was subsequently transferred to a beaker, and finally allowed to stand (i.e. be airdried) in fume hood at $50{ }^{\circ} \mathrm{C}$ (oil bath) for at least $1.5 \mathrm{~h}$, followed by vacuum drying at $80{ }^{\circ} \mathrm{C}$ for at least $48 \mathrm{~h}$, to thoroughly remove unreacted HMDS molecules, any by-product(s), etc. An HMDS-modified nanosilica was thus obtained, still as a white powder.

\section{Fourier transform infrared (FTIR) spectroscopy}

An FTIR spectrometer (Bruker, Tensor II) was used to collect the absorption spectra, of a $4 \mathrm{~cm}^{-1}$ resolution at room temperature (RT), of EP44, TMDMS, the TMDMS-grafted EP44, HMDS, the nanosilica, and the HMDS-modified nanosilica, based on 16 scans between $4000-600 \mathrm{~cm}^{-1}$ in the attenuated total reflection (ATR) mode for the former four liquids and 24 scans between 4000-400 $\mathrm{cm}^{-1}$ in the transmission mode for the latter two solids. For the runs, a drop ( $15-20 \mathrm{mg}$ ) of each of the liquids samples was pipetted onto the center of the ATR attachment, while the solids samples were prepared as $\mathrm{KBr}$ pellets by grinding together, using a pestle and mortar, $\sim 2.0 \mathrm{mg}$ of either solid powder and $\sim 50 \mathrm{mg}$ of $\mathrm{KBr}$ dried with an infrared baking lamp, followed by compression of the mixture powder on a hydraulic press. Prior to the spectroscopic measurements, samples of EP44, the TMDMS-grafted EP44, and the HMDSmodified nanosilica were all dried in vacuo at $80{ }^{\circ} \mathrm{C}$ for at least $48 \mathrm{~h}$, and that of the nanosilica was vacuum-dried at $150{ }^{\circ} \mathrm{C}$ for $5 \mathrm{~h}$. 


\section{${ }^{1} \mathrm{H}$ nuclear magnetic resonance (NMR) spectroscopy}

An NMR spectrometer (Varian, INOVA $600 \mathrm{MHz}$ ) was employed to collect the ${ }^{1} \mathrm{H}$ spectra of EP44, TMDMS, and the TMDMS-grafted EP44 with respect to a tetramethylsilane internal standard. For the runs, two drops ( $\sim 30-40 \mathrm{mg}$ ) of each of the liquid compounds fully were dissolved in $1.0 \mathrm{~g}$ of deuterated chloroform in an NMR tube to form a uniform solution sample of $\sim 30-40 \mathrm{mg} \mathrm{g}^{-1}$. The spectra of the ${ }^{1} \mathrm{H}$ peak chemical shifts $(\delta$ 's) of the solution samples all were simulated using Chemdraw to aid the assignment analysis.

\section{Thermogravimetric analysis (TGA)}

A thermogravimetric-differential-thermal simultaneous analyzer (TA Instruments, SDT Q600) was applied to study the weight decreasing behaviour, until $800{ }^{\circ} \mathrm{C}$, of the HMDS-modified nanosilica against that of the nanosilica. For the runs, $\sim 5.0 \mathrm{mg}$ of either sample of the nanosilica powder and the HMDSmodified nanosilica powder, upon vacuum drying at $150{ }^{\circ} \mathrm{C}$ for $5 \mathrm{~h}$ and at $80{ }^{\circ} \mathrm{C}$ for at least $48 \mathrm{~h}$ respectively, was placed into an alumina crucible, which was then loaded onto the alumina sample tray of the internal balance. TGA thermograms were recorded from RT to $800{ }^{\circ} \mathrm{C}$ at a heating rate of $10^{\circ} \mathrm{C} \mathrm{min}{ }^{-1}$ under a nitrogen atmosphere (i.e. purge gas) of $100 \mathrm{~mL} \mathrm{~min}^{-1}$.

\section{Surface treatment of a tinplate substrate with alkaline solution}

The as-purchased tinplate substrates $\left(120 \times 50 \times 2.8 \mathrm{~mm}^{3}\right)$ were first polished in turn by abrasive Sand-papers \#240, \#600 and \#1000 of increasing meshes, then degreased in RT acetone for $5 \mathrm{~min}$, subsequently treated in a $60{ }^{\circ} \mathrm{C}, 2 \mathrm{wt} \% \mathrm{NaOH}$ aqueous solution for $2 \mathrm{~min}$, and finally vacuum-dried at $100{ }^{\circ} \mathrm{C}$ for at least $48 \mathrm{~h}$.

\section{Preparation of epoxy resin based coatings against the surface- treated tinplate substrate}

First, the formulae of the six coating compositions investigated are listed in Table 1, which, respectively, were based on EP44, the TMDMS-grafted EP44, and the mixtures of the TMDMSgrafted EP44 and different contents (0.5, 1, 3 and 5 wt $\%)$ of the HMDS-modified nanosilica powder, in the presence of an amino-terminated polyamide-based curing agent (i.e. G650) and a mixed solvent of $n$-butanol/xylene $(7 / 3 \mathrm{w} / \mathrm{w})$. In each composition of Table 1 , the loading levels of all the other ingredients relative to that of the base resin (EP44 or TMDMS-grafted EP44) were determined according to the rules indicated in the footnote to Table 1.

Then, the mixtures of the TMDMS-grafted EP44 and the different contents of the HMDS-modified nanosilica were prepared as follows. As illustrated in Scheme 3(1)-(4), predetermined amounts, i.e. $0.084,0.168,0.515$ and $0.876 \mathrm{~g}$ corresponding to $0.5,1,3$ and $5 \mathrm{wt} \%$ respectively ( $c f$. Table 1$)$, of the HMDS-modified nanosilica powder each were added into a $50 \mathrm{~mL}$ beaker along with $\sim 40 \mathrm{~g}$ of acetone. The suspension was sonicated at a power of $270 \mathrm{~W}$ for 10 min under the protection of an iced water bath to form an organosol, which was then poured into a $250 \mathrm{~mL}$ beaker containing $9.00 \mathrm{~g}$ (according to Table 1(c-f) of the TMDMS-grafted EP44. The pristine mixture was stirred at RT for $\sim 1 \mathrm{~min}$, subsequently airdried in fume hood at RT for at least $6 \mathrm{~h}$, and finally vacuumdried at $80{ }^{\circ} \mathrm{C}$ overnight to obtain the acetone-free mixture of the TMDMS-grafted EP44 and the HMDS-modified nanosilica.

Finally, the application and cure of the six coating compositions to the surface-treated tinplate substrate was completed. As illustrated in Scheme 3(4)-(7), $7.64 \mathrm{~g}$ of the curing agent G650 and $8.40 \mathrm{~g}$ of the $n$-butanol/xylene $(7 / 3 \mathrm{w} / \mathrm{w})$ mixed solvent, read from Table $1(\mathrm{c}-\mathrm{f})$, together were stirred with each of the obtained (TMDMS-grafted EP44)/(HMDS-modified nanosilica) mixtures, in the beaker, at RT for $\sim 5 \mathrm{~min}$ to form a curable coating composition (basically solution), which was then applied uniformly to the surface-treated tinplate substrate at RT, subsequently cured in fume hood at RT for 7 days, and finally postcured in vacuo, through a ramp from RT to $70{ }^{\circ} \mathrm{C}$ within $\sim 1 \mathrm{~h}$ followed by a hold there for $3 \mathrm{~h}$, to obtain a composite coating completely cured against the tinplate

Table 1 Formulae of the coating compositions based on (a) an epoxy resin (EPR) of bisphenol-A origin with an epoxy value of 0.440, i.e. EP44, (b) a 3,3,3-trifluoropropylmethyldimethoxysilane (TMDMS) grafted EP44, and the mixtures of the TMDMS-grafted EP44 filled with different contents (wt\%): (c) 0.5; (d) 1; (e) 3; (f) 5 of 1,1,1,3,3,3-hexamethyldisilazane (HMDS) modified nanosilica powder, in the presence of an amino-terminated polyamide-based curing agent (i.e. G650) and a mixed solvent of $n$-butanol/xylene $(7 / 3 \mathrm{w} / \mathrm{w})^{a}$

\begin{tabular}{|c|c|c|c|c|c|c|}
\hline $\begin{array}{l}\text { EPR-based coating } \\
\text { no. }\end{array}$ & EP44 (g) & $\begin{array}{l}\text { TMDMS-grafted } \\
\text { EP44 (g) }\end{array}$ & $\begin{array}{l}\text { HMDS-modified } \\
\text { nanosilica }(\mathrm{g})\end{array}$ & $\begin{array}{l}\text { Curing agent } \\
\text { G650 }(\mathrm{g})\end{array}$ & $\begin{array}{l}n \text {-Butanol } \\
\text { (g) }\end{array}$ & $\begin{array}{l}\text { Xylene } \\
\text { (g) }\end{array}$ \\
\hline (a) & 9.00 & 0 & 0 & 9.00 & 5.88 & 2.52 \\
\hline (c) & 0 & 9.00 & 0.084 & 7.64 & 5.88 & 2.52 \\
\hline (d) & 0 & 9.00 & 0.168 & 7.64 & 5.88 & 2.52 \\
\hline (e) & 0 & 9.00 & 0.515 & 7.64 & 5.88 & 2.52 \\
\hline
\end{tabular}

${ }^{a}$ In formulae a-f, the mass ( $8.40 \mathrm{~g}$ ) of the mixed $n$-butanol/xylene solvent was determined according to its mass ratio to the (TMDMS-grafted) EP44 $(9.00 \mathrm{~g})$ of $1 / 1.07$, which was found to be optimal in the coating viscosity for the thickness uniformity of the coatings applied to the tinplate substrate; the mass of the G650 was equal to that $(9.00 \mathrm{~g}$ ) of EP44 (in Formula a), or to that (7.64 g) of the EP44 moieties of the TMDMS-grafted EP44 (in formulae b-f) under the assumption that the EP44 hydroxyls $100 \%$ were consumed upon the graft reaction; and, in formulae c-f, the contents $(0.5,1,3$ and $5 \mathrm{wt} \%$ corresponding to $0.084,0.168,0.515$ and $0.876 \mathrm{~g}$, respectively) of the HMDS-modified nanosilica each were determined as the ratio of its mass to the total mass of the TMDMS-grafted EP44, the G650, and itself. 


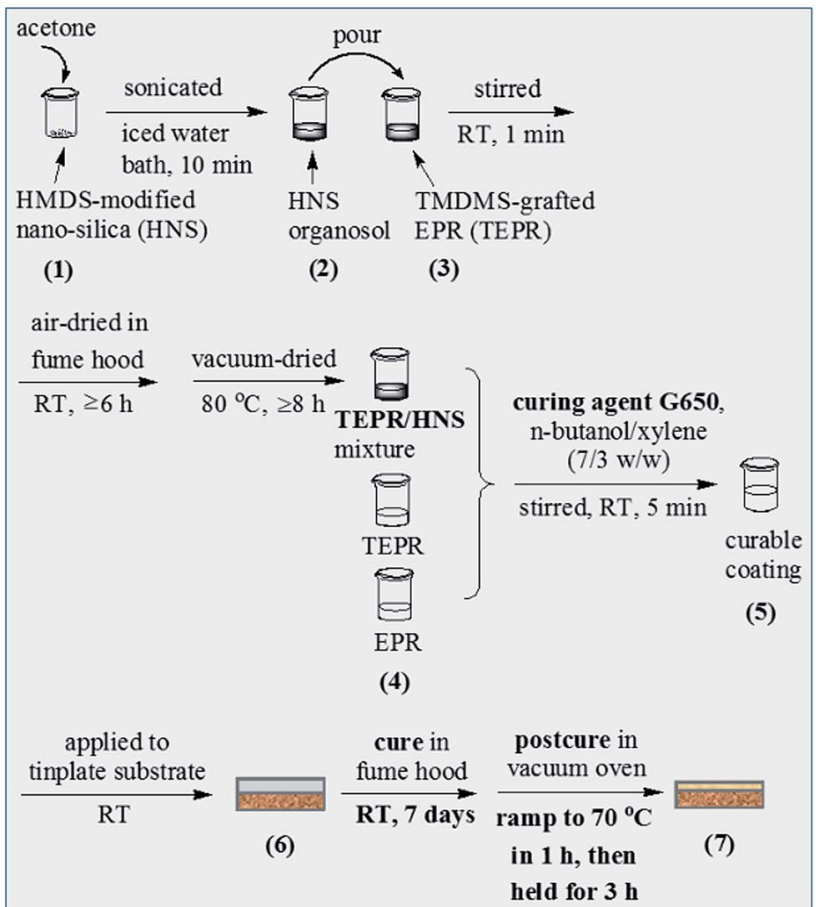

Scheme 3 Illustration of the preparation and application process, to a $2 \mathrm{wt} \% \mathrm{NaOH}$ solution treated tinplate substrate, of the corrosion preventive coating based on an epoxy resin (EPR) of bisphenol-A origin with an epoxy value of 0.440 , i.e. EP44, on a 3,3,3-trifluoropropylmethyldimethoxysilane (TMDMS) grafted EP44, or on the mixtures of the TMDMS-grafted EP44 and a 1,1,1,3,3,3-hexamethyldisilazane (HMDS) modified nanosilica, in the presence of an amino-terminated polyamide-based curing agent (i.e. G650) and a mixed solvent of $n$-butanol/xylene $(7 / 3 \mathrm{w} / \mathrm{w})$.

substrate. This procedure (i.e. Scheme 3(4)-(7)) was repeated for the preparation of two base resin coatings, respectively, by curing the solutions of EP44 and the TMDMS-grafted EP44 in the mixed solvent with the G650 present, according to the formulae of Table 1(a and $b$ ). In the cure, the formed crosslinked structure and typical cross-linking reaction, respectively, presumably are represented in Scheme $4 \mathrm{a}(1)$ and b, while those during the postcure in Scheme $4 \mathrm{a}(2)$ and c respectively. Measured by a coating pachymeter (ARCO Science \& Technology Ltd., China, Smart Sensor® AR932), the thicknesses of all the coatings, onto the substrate, made from the six compositions were maintained at $52 \pm 4 \mu \mathrm{m}$, which were then stored in a desiccator for later use.

\section{Contact angle measurements}

The contact angles $\left(\theta^{\prime} \mathrm{s}\right)$, at $\mathrm{RT}\left(\sim 25^{\circ} \mathrm{C}\right)$, of water against the six compositions of coatings fully cured onto the tinplate substrate were measured on an optical contact angle goniometer (Shanghai Zhongchen Digital Technic Apparatus Co., Ltd., China, JC-2000D). First, a syringe filled with $100 \mu \mathrm{L}$ of distilled water was made to slowly extrude a pendant water droplet of $\sim 0.1 \mu \mathrm{L}$ attached to its needle tip. Then, the stage loaded with a tinplate coating sample, below the tip, was raised to allow the surface of the coating to contact the droplet, and subsequently

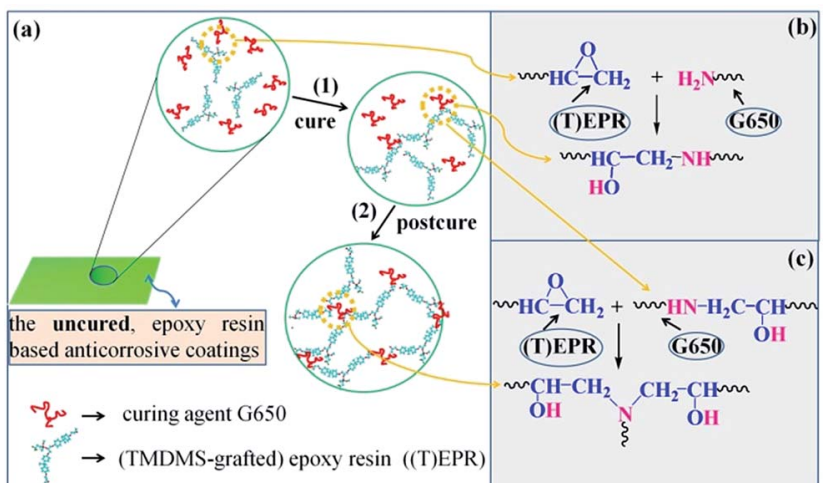

Scheme 4 (a) Representation of the cross-linked structures formed during the curing process of a (3,3,3-trifluoropropylmethyldimethoxysilane (TMDMS) grafted) epoxy resin (EPR) with an amino-terminated polyamide-based curing agent (i.e. G650): (1) cure at room temperature for 7 days to form $V$-shaped cross-links; (2) subsequent postcure via a ramp from room temperature to $70{ }^{\circ} \mathrm{C}$ in $1 \mathrm{~h}$ followed by a hold there for $3 \mathrm{~h}$ further to form $\mathrm{X}$ shaped cross-links. (b) Schematic of the typical cross-linking reaction during the cure (i.e. step 1 in (a)), in which the epoxide groups of the (TMDMS-grafted) EPR react with the amino groups of the G650 via ring-opening addition to produce hydroxy and imino groups. (c) Schematic of the typical cross-linking reaction during the postcure (i.e. step 2 in (a)), in which the epoxide groups of the (TMDMS-grafted) EPR react with the imino groups of the G650 via ring-opening addition to produce hydroxy and nitrilo groups.

lowered slowly to detach the droplet from the tip, thus forming a sessile water droplet onto the coating. After aligning the baseline right with the coating surface, a software program of digital video image processing (JC-2000D) was used to analyse the droplet contour, from which the water $\theta$ of the coating was calculated. For each composition of coating, at least three parallel samples were measured to obtain three $\theta$ values, respectively, from which the median value was taken as the $\theta$ for analysis.

\section{Electrochemical impedance spectroscopy (EIS)}

A sealant for insulation of the coatings cured onto the tinplate substrate was prepared from a curable EP44/G650 (1/1 w/w) composition as follows: $20 \mathrm{~g}$ of the composition in a $250 \mathrm{~mL}$ beaker was stirred for $2 \mathrm{~min}$, then deaerated in vacuo for $30 \mathrm{~min}$, and subsequently allowed to stand (i.e. pre-cure) overnight, all at RT, to ensure that the flowability of the gelling composition (i.e. sealant) considerably was reduced compared with the pristine composition. Immediately, the PVC tubes (of $40 \mathrm{~mm}$ in outer diameter, $36.6 \mathrm{~mm}$ in inner diameter, and $50 \mathrm{~mm}$ in length) each, by their end cross sections, were dipped in a predetermined dosage of the sealant and then attached (i.e. glued) to the surface of each of the tinplate coatings, which was followed by pipetting an appropriate amount of the sealant around the seam between the coating and the PVC tube. At last, the PVC tube-attached coatings (i.e. EIS specimens) were cured (with respect to the sealant) at RT for at least $48 \mathrm{~h}$ and then stored in a desiccator at all times prior to EIS experiments.

The anticorrosive performance of the six compositions of coatings was therefore measured at RT using EIS, a test mode of 
an electrochemical workstation (CH Instruments, Inc., China, CHI660E). Each of the (PVC tube-attached) coating specimens was immersed into an $\mathrm{NaCl}$ aqueous solution of $3.5 \mathrm{wt} \%$ at RT $\left(\sim 25{ }^{\circ} \mathrm{C}\right.$ ) by adding $\sim 47 \mathrm{~mL}$ of the solution into the tube that was positioned vertically against the levelled coating, with an immersion area of $\sim 10.5 \mathrm{~cm}^{2}$ calculated from the tube innerdiameter of $36.6 \mathrm{~mm}$. The EIS runs of each coating specimen across a frequency range of $10^{5}$ to $10^{-2} \mathrm{~Hz}$ were conducted, respectively, upon its increasing immersion times of 1, 24, 240, 480, 720 and $960 \mathrm{~h}$. For the experiments, a three-electrode arrangement was used, which comprised the tinplate substrate working electrode, a reference electrode of $\mathrm{Ag} / \mathrm{AgCl}$ in saturated $\mathrm{KCl}$ aqueous solution at RT, and a counter electrode of platinum; the latter two electrodes were both immersed into the $\mathrm{NaCl}$ solution of the coating specimens, with the reference electrode bridged by another electrolytic aqueous solution of saturated $\mathrm{KCl}$ at RT. Each composition of coating was measured using five coating specimens, from which the EIS spectra (i.e. Bode plots) upon the increasing immersion times of the specimen having a median spectrum upon $1 \mathrm{~h}$ of immersion were taken as the experimental data for analysis with the aid of the Zsimpwin software.

\section{Results and discussion}

Evidence for successful grafting of 3,3,3trifluoropropylmethyldimethoxysilane onto epoxy resin molecules

Fig. 1 shows the FTIR spectra of (1) EP44, (2) TMDMS, and (3) the TMDMS-grafted EP44 in the wavenumber ranges of (a) $4000-2400 \mathrm{~cm}^{-1}$ and (b) $2400-600 \mathrm{~cm}^{-1}$. In spectrum 2 of TMDMS, the absorption peaks (and shoulder) at 2967, 2915, 2946,2841 and $1080 \mathrm{~cm}^{-1}$, respectively, were attributable to the $\mathrm{C}-\mathrm{H}$ antisymmetric and symmetric stretches of its $-\mathrm{CH}_{3}$ groups, the $\mathrm{C}-\mathrm{H}$ antisymmetric and symmetric stretches of its $-\mathrm{CH}_{2}-$ groups, and the $\mathrm{C}-\mathrm{O}$ stretching of its $-\mathrm{OCH}_{3}$ groups. Since the $827 \mathrm{~cm}^{-1}$ absorption band, ascribed to the $\mathrm{C}-\mathrm{H}$ out-of-plane bending of the bisphenol-A phenylenes, was supposed to be unchanged in absorbance upon the possible graft reaction, Spectra 1 and 3, respectively, of EP44 and the TMDMS-grafted EP44 both were normalised with respect to it for a typical comparison. In spectrum 1 of EP44: the bands at 2965, 2871, 2926, 2838, 1230 and $1031 \mathrm{~cm}^{-1}$, respectively, were assigned to the $\mathrm{C}-\mathrm{H}$ antisymmetric and symmetric stretches of its $-\mathrm{CH}_{3}$ groups, the $\mathrm{C}-\mathrm{H}$ antisymmetric and symmetric stretches of the $-\mathrm{CH}_{2}-$ neighbouring its epoxide groups, and the $\mathrm{C}-\mathrm{O}-\mathrm{C}$ antisymmetric and symmetric stretches of its methyleneoxyphenylene groups; those at 1606, 1580 and $1507 \mathrm{~cm}^{-1}$ probably were attributed to the skeletal vibrations of its phenylenes; those at 1384 and $1364 \mathrm{~cm}^{-1}$ were the split, weak double bands from the $\mathrm{C}-\mathrm{H}$ symmetric bending of its $-\mathrm{C}\left(\mathrm{CH}_{3}\right)_{2}-$ groups; and that at $911 \mathrm{~cm}^{-1}$ likely was characteristic of the $\mathrm{C}-\mathrm{O}$ deformational vibration of its epoxide groups. All these bands (including that at $827 \mathrm{~cm}^{-1}$ ) appeared as well in spectrum 3 of the TMDMSgrafted EP44, at exactly the same wavenumbers and comparable absorbances, indicating that the graft reaction, if any, might not involve the aforementioned groups of EP44.
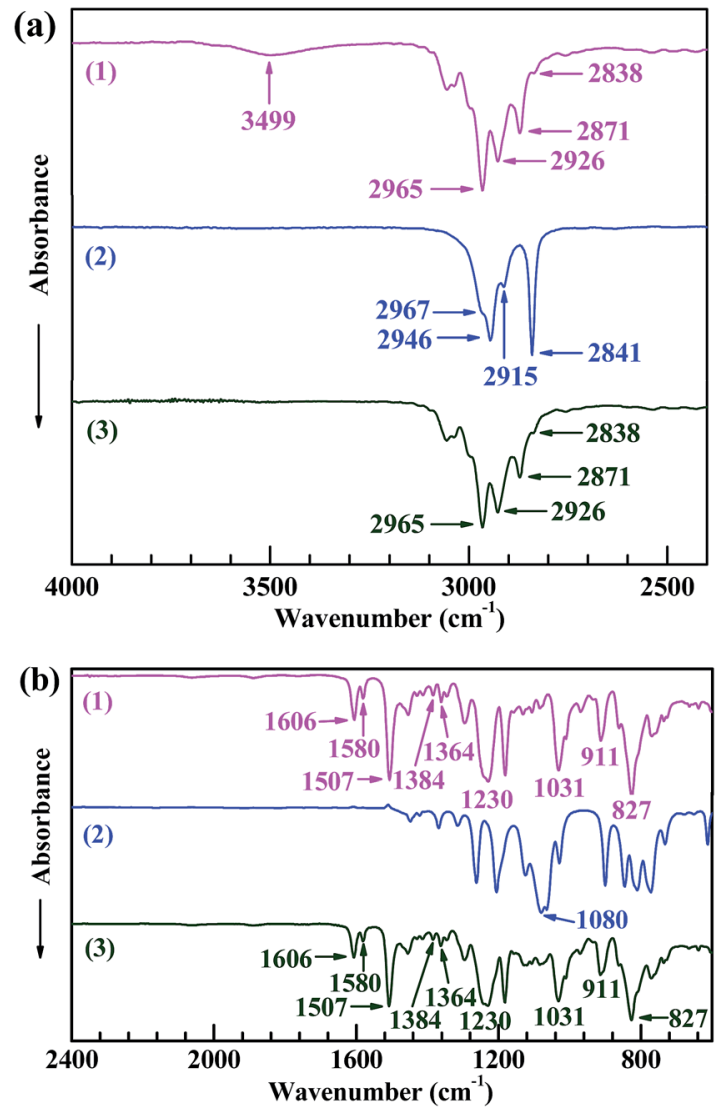

Fig. 1 Fourier transform infrared (FTIR) absorption spectra of (1) a bisphenol-A based epoxy resin of an epoxy value of 0.440 (i.e. EP44), (2) 3,3,3-trifluoropropylmethyldimethoxysilane (TMDMS), and (3) a TMDMS-grafted EP44, (a) between 4000 and $2400 \mathrm{~cm}^{-1}$ and (b) between 2400 and $600 \mathrm{~cm}^{-1}$, at room temperature in the attenuated total reflection (ATR) mode. In graph a, the $3499 \mathrm{~cm}^{-1}$ absorption band in spectrum 1 characteristic of the $\mathrm{O}-\mathrm{H}$ stretching of the $-\mathrm{OH}$ groups present in EP44 molecules nearly disappears in spectrum 3 of the TMDMS-grafted EP44 molecules, suggesting the significant occurrence of a graft reaction that consumes dramatically the EP44 $-\mathrm{OH}$ groups.

In Fig. 1a, the $3499 \mathrm{~cm}^{-1}$ absorption band in spectrum 1 of EP44 molecules characterised the $\mathrm{O}-\mathrm{H}$ stretching of their $-\mathrm{OH}$ groups, which, however, nearly disappeared in spectrum 3 of the TMDMS-grafted EP44 molecules, revealing a dramatic consumption of the -OH groups of EP44 through its significant graft modification; in other words, the $-\mathrm{OH}$ groups participated as a leading role in the graft reaction. It is worth mentioning that, since both EP44 and the TMDMS-grafted EP44 were well dried (in vacuo at $80{ }^{\circ} \mathrm{C}$ for at least $48 \mathrm{~h}$ ) prior to their FTIR analysis, the peak disappearance should result largely from a sharp decrease in the $-\mathrm{OH}$ concentration, but not primarily from a considerably decreased content of adsorbed water. Therefore, under the anhydrous, DBTDL-catalytic conditions, the $-\mathrm{OCH}_{3}$ groups of TMDMS, most reactive among all its groups, presumably were reacted markedly with the -OH groups of EP44, via transesterification (i.e. alcoholysis), to produce a TMDMS-grafted EP44 plus a methanol by-product, as schematically shown in Scheme 1b; mechanistically, the -OH groups 
of EP44 molecules were substituted electrophilically by TMDMS molecules under anhydrous catalysis of DBTDL. These FTIR results agree with other reports ${ }^{\mathbf{1 4 , 2 3}}$ in the literature and confirm that TMDMS successfully was grafted to EP44 molecules, to a significant extent, at the -OH group sites of EP44.

Fig. 2 shows the ${ }^{1} \mathrm{H}$ NMR spectra of (a, d) EP44, (b, e) TMDMS, and (c, f) the TMDMS-grafted EP44. The supposed $\mathrm{H}$ atoms of EP44, TMDMS and the TMDMS-grafted EP44 are numbered in Fig. 2(d-f), respectively, to which the ${ }^{1} \mathrm{H}$-peak $\delta$ values in Fig. $2(\mathrm{a}-\mathrm{c})$ were assigned as given in Table 2. In Fig. 2a of EP44 ( $c f$. Fig. 2d): the peaks of the $\mathrm{H}$ atoms of its epoxide $\mathrm{CH} \equiv$ (no. 2), phenylenes (no. 4 and 5) and $-\mathrm{C}\left(\mathrm{CH}_{3}\right)_{2}-$ (no. 6) appeared at $\delta$ values of 3.28, 7.11, 6.81 and $1.61 \mathrm{ppm}$, respectively; the peak of the $\mathrm{H}$ atoms of its epoxide $-\mathrm{CH}_{2}-($ no. 1)
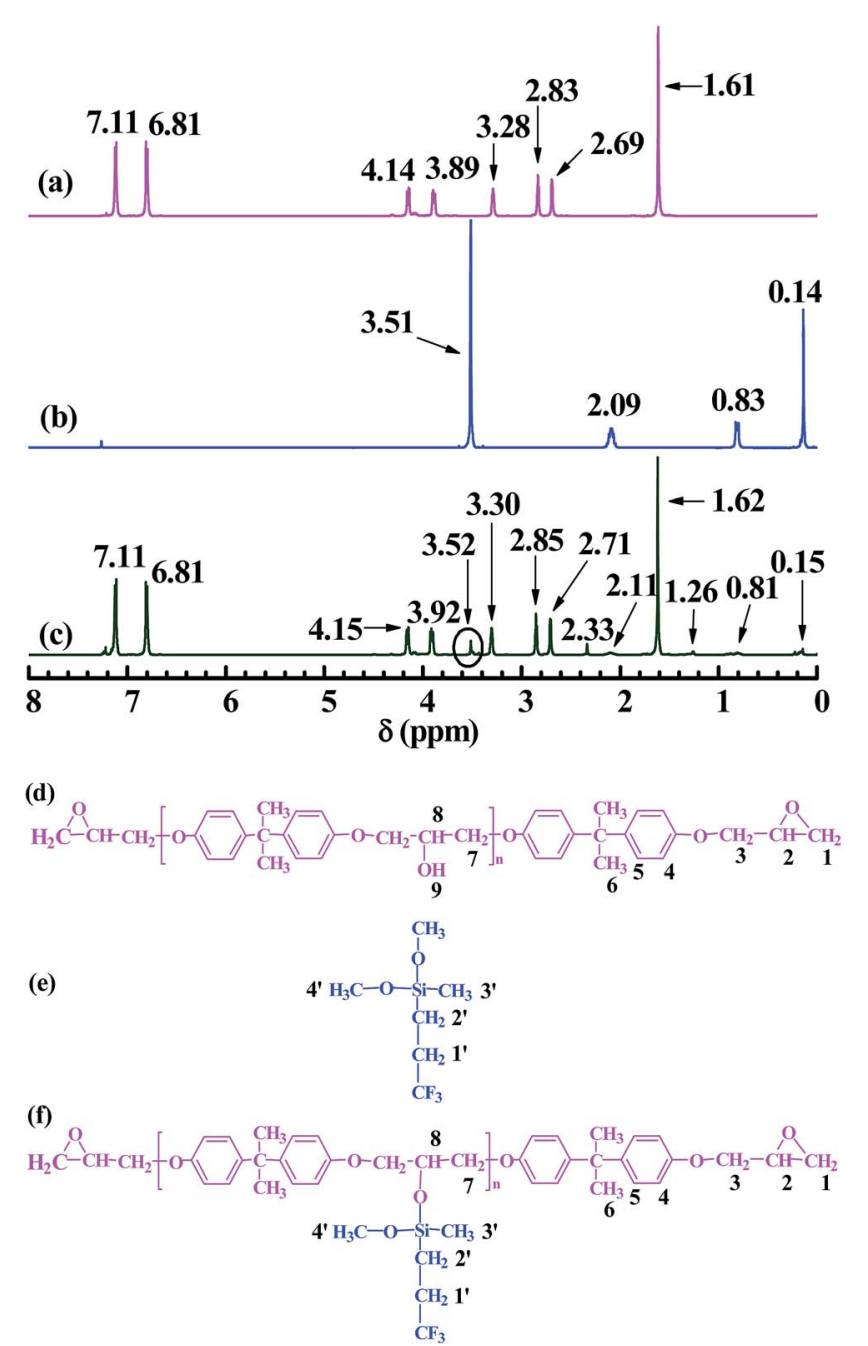

Fig. $2{ }^{1} \mathrm{H}$ nuclear magnetic resonance (NMR) spectra of (a) a bisphenol-A based epoxy resin of an epoxy value of 0.440 (i.e. EP44), (b) 3,3,3-trifluoropropylmethyldimethoxysilane (TMDMS), and (c) a TMDMS-grafted EP44, respectively, for $\mathrm{H}$-atoms no. 1-6 shown in (d), $1^{\prime}-4^{\prime}$ shown in (e), and $1-8$ and $1^{\prime}-4^{\prime}$ shown in (f); the assignments of (a) to (d), (b) to (e), and (c) to (f) are given in Table 2, where the chemical shift $(\delta)$ values of $2.09,0.83,0.14$ and $3.51 \mathrm{ppm}$, respectively, for $\mathrm{H}$-atoms no. $1^{\prime}-4^{\prime}$ of TMDMS molecules all are present in the ${ }^{1} \mathrm{H}$ NMR spectrum of the TMDMS-grafted EP44 molecules, confirming a successful grafting of TMDMS onto EP44. split in two at 2.69 and $2.83 \mathrm{ppm}$, and that of the $-\mathrm{CH}_{2}-$ neighbouring its epoxides (no. 3) at 3.89 and $4.14 \mathrm{ppm}$, either of which might be caused by a variation in the respective steric configuration; ${ }^{31}$ the peak of its $-\mathrm{OH}$ hydrogens (no. 9) was missing presumably due to their high activity, which possibly repressed as well the peaks of their neighbouring $\mathrm{H}$ atoms (no. 7 and 8). In Fig. 2b of TMDMS ( $c f$. Fig. 2e), the peaks of the $\mathrm{H}$ atoms of its $\mathrm{CF}_{3}-\mathrm{CH}_{2}-\left(\right.$ no. $\left.1^{\prime}\right),-\mathrm{CH}_{2}-\mathrm{Si} \equiv$ (no. $2^{\prime}$ ), $\equiv \mathrm{Si}-\mathrm{CH}_{3}$ (no. $\left.3^{\prime}\right)$ and $-\mathrm{Si}\left(\mathrm{OCH}_{3}\right)_{2}-\left(\right.$ no. $\left.4^{\prime}\right)$, respectively, occurred at 2.09, 0.83, 0.14 and $3.51 \mathrm{ppm}$. As shown in Fig. 2c ( $c f$. Fig. 2f): the spectrum of the TMDMS-grafted EP44 completely resembled that of EP44 (Fig. 2a) with the additional $\mathrm{H}$-atom peaks at 2.11, 0.81, 0.15 and $3.52 \mathrm{ppm}$, which obviously were attributed, respectively, to the $\mathrm{CF}_{3}-\mathrm{CH}_{2}-\left(\right.$ no. $\left.1^{\prime}\right),-\mathrm{CH}_{2}-\mathrm{Si} \equiv\left(\right.$ no. $\left.2^{\prime}\right), \equiv \mathrm{Si}-\mathrm{CH}_{3}$ (no. $3^{\prime}$ ) and $\equiv \mathrm{Si}-\mathrm{O}-\mathrm{CH}_{3}$ (no. $4^{\prime}$ ) groups of the TMDMS graft ( $c f$. Fig. $2 \mathrm{~b}$ and Table 2), despite their sharply weakened intensities due to the significantly decreased group concentrations (note that $n \sim 0.4$ in Fig. 2f, for EP44); moreover, the peaks of H-atoms no. 7 and 8 of EP44 moieties emerged upon the graft reaction at 2.33 and $1.26 \mathrm{ppm}$, respectively, suggesting a pronounced elimination of the active -OH hydrogens (no. 9 in Fig. 2d) of EP44 that released the NMR of their neighbouring $\mathrm{H}$ atoms. In addition to the above FTIR data, these NMR results further corroborate that TMDMS indeed was grafted significantly onto EP44 molecules, likely through a transesterification (i.e. alcoholysis) reaction under anhydrous, DBTDL catalyst conditions between the $-\mathrm{OCH}_{3}$ groups of TMDMS and the $-\mathrm{OH}$ groups of EP44, as schematically shown in Scheme 1 b or Fig. 2(d-f). Particularly worth noting is that the TMDMS-grafted EP44 still had the $\mathrm{H}$-atom (no. $4^{\prime}$ ) peak of the $-\mathrm{OCH}_{3}$ groups at $3.52 \mathrm{ppm}$ (Fig. 2c

Table 2 Chemical shift $(\delta)$ values of the ${ }^{1} \mathrm{H}$ nuclear magnetic resonance (NMR) peaks given in Fig. $2(a-c)$, respectively, assigned to the $\mathrm{H}$ atoms, of a bisphenol-A based epoxy resin of an epoxy value of 0.440 (i.e. EP44), 3,3,3-trifluoropropylmethyldimethoxysilane (TMDMS), and a TMDMS-grafted EP44, whose no. in the molecular structures are shown in Fig. 2(d-f)

\begin{tabular}{llll}
\hline & \multicolumn{1}{l}{$\delta(\mathrm{ppm})$} & & \\
\cline { 2 - 4 } H atom no. & EP44 & TMDMS & TMDMS-grafted EP44 \\
\hline 1 & $2.69,2.83$ & $-^{b}$ & $2.71,2.85$ \\
2 & 3.28 & $-^{b}$ & 3.30 \\
3 & $3.89,4.14$ & $-^{b}$ & $3.92,4.15$ \\
4 & 7.11 & $-^{b}$ & 7.11 \\
5 & 6.81 & $-^{b}$ & 6.81 \\
6 & 1.61 & $-^{b}$ & 1.62 \\
7 & $-^{a}$ & $-{ }^{b}$ & 2.33 \\
8 & $-^{a}$ & $-{ }^{b}$ & 1.26 \\
9 & $-^{b}$ & $-{ }^{b}$ & $-{ }^{b}$ \\
$1^{\prime}$ & $-^{b}$ & 2.09 & 2.11 \\
$2^{\prime}$ & $-{ }^{b}$ & 0.83 & 0.81 \\
$3^{\prime}$ & $-{ }^{b}$ & 0.14 & 0.15 \\
$4^{\prime}$ & & 3.51 & 3.52
\end{tabular}

${ }^{a}$ Owing to the high activity of the EP44-OH groups, the $\delta$ values of their $\mathrm{H}$ atoms (i.e. $\mathrm{H}$-atoms no. 9) and neighbouring $\mathrm{H}$ atoms (i.e. $\mathrm{H}$-atoms no. 7 and 8) are not subject to detection. ${ }^{b}$ These $\mathrm{H}$ atoms do not belong to EP44, TMDMS and the TMDMS-grafted EP44 molecules, respectively. 
vs. f), disclosing that a single TMDMS molecule reacted with the EP44 -OH largely by only one $-\mathrm{OCH}_{3}$, with the other $-\mathrm{OCH}_{3}$ remaining in the TMDMS graft possibly due to a steric hindrance effect.

\section{Confirmation of successful modification of nanosilica with 1,1,1,3,3,3-hexamethyldisilazane}

The reaction between the nanosilica and HMDS was characterised by FTIR spectroscopy as shown in Fig. 3. In spectrum 2 of HMDS, the absorption bands at 3381, 2955, 2898, 1250 and $834 \mathrm{~cm}^{-1}$, respectively, were ascribed to its $\mathrm{N}-\mathrm{H}$ stretching, the $\mathrm{C}-\mathrm{H}$ antisymmetric and symmetric stretches of its $-\mathrm{CH}_{3}$ groups, and the $\mathrm{Si}-\mathrm{C}$ deformation and rocking vibrations of its $-\mathrm{Si}\left(\mathrm{CH}_{3}\right)_{3}$ groups. $^{32-34}$ To make a typical comparison, both spectra 1 and 3 of the nanosilica and HMDS-modified nanosilica, respectively, were normalised with respect to their band at $473 \mathrm{~cm}^{-1}$, which, likely characteristic of the Si-O-Si bending of the silica gel networks, presumably was unchanged in absorbance upon the surface modification. Thus, the 1108 and $803 \mathrm{~cm}^{-1}$ bands, respectively, from the $\mathrm{Si}-\mathrm{O}-\mathrm{Si}$ antisymmetric and symmetric stretches of the silica gel networks ${ }^{35}$ both changed little as well in absorbance despite the modification of the nanosilica surfaces (spectrum 1 vs. 3). However, the $3420 \mathrm{~cm}^{-1}$ band in spectrum 3 of the HMDS-modified nanosilica, which was attributed to an $\mathrm{O}-\mathrm{H}$ stretching, weakened considerably compared with that in spectrum 1 of the nanosilica; as both the samples were well dried (in vacuo, at $80^{\circ} \mathrm{C}$ for at least $48 \mathrm{~h}$ and at $150^{\circ} \mathrm{C}$ for $5 \mathrm{~h}$, respectively) prior to the FTIR

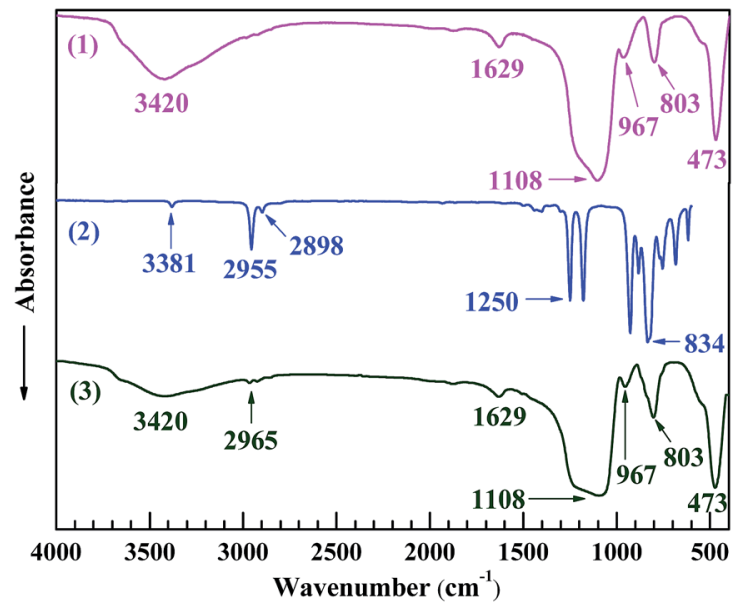

Fig. 3 Fourier transform infrared (FTIR) absorption spectra of (1) a nanosilica powder in the transmission mode, (2) 1,1,1,3,3,3-hexamethyldisilazane (HMDS) in the attenuated total reflection (ATR) mode, and (3) an HMDS-modified nanosilica powder in the transmission mode, between 4000 and $400 \mathrm{~cm}^{-1}$ wavenumbers at room temperature. In spectrum 3 of the HMDS-modified nanosilica powder, the absorption band at $3420 \mathrm{~cm}^{-1}$ characteristic of the $\mathrm{O}-\mathrm{H}$ stretching of its surface $-\mathrm{OH}$ groups weakens significantly compared with that in spectrum 1 of the nanosilica powder, which, along with the emergence of a new band at $2965 \mathrm{~cm}^{-1}$ characteristic of the $\mathrm{C}-\mathrm{H}$ antisymmetric stretching of $-\mathrm{CH}_{3}$ groups, indicates the occurrence of a significant surface modification of the nanosilica powder with HMDS that converts $-\mathrm{OH}$ to $-\mathrm{CH}_{3}$ groups. runs, this band weakening essentially should originate from a significant decrease in the surface $-\mathrm{OH}$ concentration upon the modification, but not from a marked reduction in the water absorption, which may be verified by the fact that the $1629 \mathrm{~cm}^{-1}$ band in spectrum 3, assigned to the $\mathrm{H}-\mathrm{O}-\mathrm{H}$ bending of the bound water, varied little in absorbance compared with that in spectrum 1. Moreover, the $967 \mathrm{~cm}^{-1}$ band presumably characterising the bending of the $\equiv \mathrm{Si}-\mathrm{OH}$ at the nanosilica surfaces weakened notably after the HMDS modification (spectrum 1 vs. 3 ), inferring again a pronounced reduction in the surface $-\mathrm{OH}$ groups. Finally, a new band at $2965 \mathrm{~cm}^{-1}$, feature of the $\mathrm{C}-\mathrm{H}$ antisymmetric stretch of $-\mathrm{CH}_{3}$, emerged, although small, in spectrum 3 of the HMDS-modified nanosilica, indicating the formation of $-\mathrm{CH}_{3}$ groups from the nanosilica and HMDS reaction. All these observations confirm the occurrence of a significant surface modification of the nanosilica with HMDS, by which a lot of $-\mathrm{CH}_{3}$ groups successfully were grafted to the nanosilica surfaces via electrophilic substitution of its surface -OH groups with the $-\mathrm{Si}\left(\mathrm{CH}_{3}\right)_{3}$ groups of HMDS, as schematically shown in Scheme $2 \mathrm{~b}$.

TGA also was used to corroborate the generation of the methyl-attached organic nanosilica from the surface modification. As shown in Fig. 4, the weight losses of both the nanosilica (curve 1) and HMDS-modified nanosilica (curve 2) occurred sequentially in two stages. ${ }^{3,36}$ The first stage occurred from RT to $100{ }^{\circ} \mathrm{C}$ due mainly to an evaporation of their bound (i.e. adsorbed) water and also possibly to a condensation reaction of the surface $\equiv \mathrm{Si}-\mathrm{OH}$ groups during their thermal aggregation to release water molecules, both of which would increase with increasing the surface - $\mathrm{OH}$ concentration. It should be noted that, although they both were well dried in vacuo (at $150{ }^{\circ} \mathrm{C}$ for $5 \mathrm{~h}$ and at $80{ }^{\circ} \mathrm{C}$ for at least $48 \mathrm{~h}$, respectively), the nanosilica

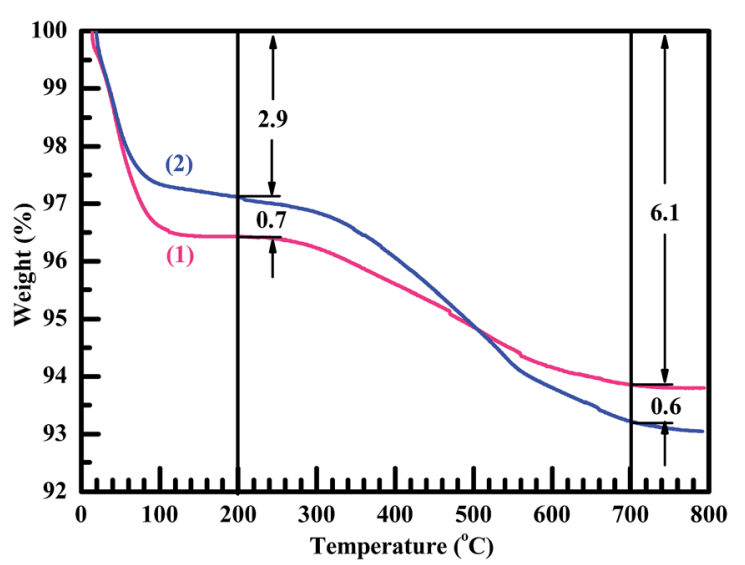

Fig. 4 (1) Thermogravimetric analysis (TGA) thermogram of a nanosilica powder against (2) that of the nanosilica powder surfacemodified with 1,1,1,3,3,3-hexamethyldisilazane (HMDS), from room temperature until $800^{\circ} \mathrm{C}$ at a rate of $10^{\circ} \mathrm{C} \mathrm{min}-1$. From 200 to $700{ }^{\circ} \mathrm{C}$, the weight loss of the HMDS-modified, organic nanosilica from the burning of its surface multiple $-\mathrm{CH}_{3}$ groups is $3.8 \%$, larger than that (2.5\%) of the nanosilica from the burning of its surface $-\mathrm{OH}$ groups, which verifies a successful grafting of $-\mathrm{CH}_{3}$ groups onto the nanosilica surfaces (i.e. conversion of the surface $-\mathrm{OH}$ to $-\mathrm{CH}_{3}$ groups) through an HMDS modification reaction. 
and modified nanosilica samples would quickly adsorb water during the short time period of after taken out of the vacuum oven and before subjected to the TGA. It is seen that the nanosilica lost its weight more than did the modified nanosilica by $\sim 0.7 \%$ in the first stage, indicating that the $-\mathrm{OH}$ concentration was decreased on the nanosilica surfaces upon its modification presumably owing to the substitution of part of the hydroxyls by the trimethylsilyl groups. The second stage occurred from 100 to $800{ }^{\circ} \mathrm{C}$ due to the burning of their surface groups: for the nanosilica, the burning primarily involved the further condensation between its surface $\equiv \mathrm{Si}-\mathrm{OH}$ groups themselves; while, for the organomodified nanosilica, the burning might cause more weight loss in that both its surface hydroxyls and organic-groups participated. In a typical 200$700{ }^{\circ} \mathrm{C}$ temperature range, it is observed from Fig. 4 that the modified nanosilica lost $3.8 \%$ of its weight, greater than $2.5 \%$ lost by the nanosilica, which verifies again the formation of grafted multiple $-\mathrm{CH}_{3}$ groups to the nanosilica surfaces by HMDS ( $c f$. Scheme $2 \mathrm{~b}$ ) that burned off more in weight than did the nanosilica hydroxyls.

\section{Hydrophobicity enhancement of epoxy resin coatings upon their grafting with a fluoroalkyl-containing organosilane}

The contact angle $(\theta)$ values of water against all the EP44-based resin coatings cured (whose compositions were given in Table $1(a-f)$ ) were measured as presented in Table 3 . It is seen that the water $\theta$ of the cured EP44 coating was $83.8^{\circ}$, revealing that it slightly was hydrophilic essentially from a concentration of hydroxy groups present. However, upon the pre-cure chemical grafting of TMDMS to EP44 molecules, the cured TMDMSgrafted EP44 coating had a considerably larger water $\theta$ of $105.5^{\circ}$, which suggests a significant enhancement of its hydrophobicity. Further, when the different contents (0.5, 1, 3 and 5 wt $\%)$ of the HMDS-modified nanosilica were added to the uncured TMDMS-grafted EP44, the water $\theta$ 's of the cured composite coatings were $101.3^{\circ}, 101.7^{\circ}, 105.9^{\circ}$ and $104.4^{\circ}$, respectively, indicating that their hydrophobicity changed little (or only slightly) with the modified nanosilica concentration despite the presence of unreacted hydrophilic hydroxyls in the added nanosilica even upon its surface modification with HMDS, as discussed in Fig. 3 and 4. From these results, it is summarised that, once grafted with TMDMS, the cured EP44 coating became significantly more hydrophobic regardless of the content of the modified nanosilica incorporated after the graft modification.

The rationale behind this hydrophobic improvement may lie in a combination of the introduction of hydrophobic trifluoropropyl groups and a decrease in the hydrophilic hydroxyl concentration both from the graft reaction, with the effect of the former predominating over that of the latter. In the molecular structure of EP44 shown in Scheme 1b or Fig. 2d, the value of $n$ is equivalent to $\sim 0.4$, while the two end epoxides, respectively, are converted to two hydroxyls upon EP44's cure. This contrast infers that, even though the EP44-TMDMS reaction was 100\% adequate, the consumed hydroxyls merely accounted for $\sim 1 / 6$ the total hydroxyls of the cured EP44; in other words, upon the grafting, the $-\mathrm{OH}$ concentration of the cured EP44 coating was reduced at the most by $17 \%$, which should contribute little to such a large increase in its hydrophobicity (i.e. water $\theta$ ) from $83.8^{\circ}$ to $105.5^{\circ}$. Instead, owing to their superhydrophobic character, the trifluoropropyl groups present in the TMDMS grafts of the cured EP44 coating likely were much more responsible for the significant enhancement of its hydrophobicity.

Table 3 Contact angles $\left(\theta^{\prime} \mathrm{s}\right)$, at room temperature $\left(\sim 25^{\circ} \mathrm{C}\right)$, of water against the corrosion protective coatings of (a) an epoxy resin (EPR) of bisphenol-A origin with an epoxy value of 0.440 (i.e. EP44), (b) a 3,3,3-trifluoropropylmethyldimethoxysilane (TMDMS) grafted EP44, and the mixtures of the TMDMS-grafted EP44 filled with different contents (wt\%): (c) 0.5; (d) 1; (e) 3; (f) 5 of 1,1,1,3,3,3-hexamethyldisilazane (HMDS) modified nanosilica powder that all are cured in an $n$-butanol/xylene $(7 / 3 \mathrm{w} / \mathrm{w})$ solution with an amino-terminated polyamide-based curing agent (i.e. G650)

\begin{tabular}{lll}
\hline EPR-based coating no. & Base resin & $\theta$ (degree) \\
(a) & TP44 & TMDMS-grafted EP44 \\
(b) & TMDMS-grafted EP44/0.5 wt\% HMDS modified nanosilica \\
(c) & TMDMS-grafted EP44/1 wt\% HMDS modified nanosilica \\
(d) & TMDMS-grafted EP44/3 wt\% HMDS modified nanosilica \\
(f) &
\end{tabular}


Improvement in the anticorrosive performance of epoxy resin coatings upon their hydrophobic modification

Although they act as a barrier protection to retard the corrosion of their underneath metallic substrate, organic polymeric coatings are far from perfect for corrosion prevention. For instance, within the EP44-based coatings investigated in this work, there might develop a number of defective pores (free volumes, voids, cracks, etc.) or channels made of them, during their preparation processes especially upon solvent evaporation, that were accessible to the corrosives (i.e. water, electrolyte $(\mathrm{NaCl})$, oxygen, other molecules and ions) by infiltration. Once the $\mathrm{NaCl}$ aqueous solution penetrated the coatings to reach the tinplate substrate, the defective pores or channels, constituting the cathodes of electrochemical galvanic cells, were populated with reactive "residents" (e.g. water, oxygen, etc.) that were subject to cathodic reduction reaction (reactions (2) and (3) below), in response to the anodic oxidation reaction (reaction (1) below) on the tinplate anode. Meanwhile, the anodic $\mathrm{Fe}^{3+}$ cations from reaction (1) migrated across the substrate-coating interface to react with the cathodic $\mathrm{OH}^{-}$anions from reactions (2) and (3), resulting in a deposition of $\mathrm{Fe}(\mathrm{OH})_{3}$ and $\mathrm{Fe}_{2} \mathrm{O}_{3}$, respectively, into the coating channels by cathodic, non-redox precipitation- and decomposition reactions (reactions (4) and (5) below).

Anodic reaction:

$$
\mathrm{Fe}-3 \mathrm{e}^{-} \rightarrow \mathrm{Fe}^{3+}
$$

Cathodic reaction:

$$
\begin{aligned}
& 2 \mathrm{H}_{2} \mathrm{O}+2 \mathrm{e}^{-} \rightarrow 2 \mathrm{OH}^{-}+\mathrm{H}_{2} \\
& \mathrm{O}_{2}+2 \mathrm{H}_{2} \mathrm{O}+4 \mathrm{e}^{-} \rightarrow 4 \mathrm{OH}^{-}
\end{aligned}
$$

Precipitation reaction:

$$
\mathrm{Fe}^{3+}+3 \mathrm{OH}^{-} \rightarrow \mathrm{Fe}(\mathrm{OH})_{3}
$$

Decomposition reaction:

$$
2 \mathrm{Fe}(\mathrm{OH})_{3} \rightarrow \mathrm{Fe}_{2} \mathrm{O}_{3}+3 \mathrm{H}_{2} \mathrm{O}
$$

The EIS Bode plots (scatter plots), i.e. plots of the logarithm of impedance magnitude $(\log |Z|) v s$. that of frequency $(\log f)$, of the six compositions of coating specimens upon their increasing immersion times $(1,24,240,480,720$ and $960 \mathrm{~h})$ in the $\mathrm{NaCl}$ solution are shown in Fig. 5 , from which their Nyquist plots (scatter plots), i.e. plots of the opposite of capacitive reactance $\left(-Z^{\prime \prime}\right) v$ s. resistance $\left(Z^{\prime}\right)$, are drawn in Fig. 6 and plots (bar plots) of the $|Z|$ at $1 \times 10^{-2} \mathrm{~Hz}$, a measure of the anticorrosive performance of the coating specimens (i.e. coatings interfacing with both the electrolyte solution and the substrate), $v s$. immersion time are made in Fig. 7a. To better understand the anticorrosion mechanism of the coatings, electrochemical equivalent-circuit models were employed to simulate the EIS experimental data. It turned out that all the Bode and Nyquist plots on 1 and $24 \mathrm{~h}$ of immersion of the six compositions of coatings were well fitted preferably by a three-impedance model, illustrated in Chart 1a, consisting of the coating resistance $\left(R_{\mathrm{c}}\right)$ and capacitance $\left(\mathrm{CPE}_{\mathrm{c}}\right)$, the charge-transfer resistance $\left(R_{\mathrm{ct}}\right)$ and double-layer capacitance $\left(\mathrm{CPE}_{\mathrm{dl}}\right)$ at the electrolytecoating interface, and the charge-transfer resistance $\left(R_{\mathrm{sf}}\right)$ and double-layer capacitance $\left(\mathrm{CPE}_{\mathrm{sf}}\right)$ at the coating-substrate interface, but not by a simple model of the $R_{\mathrm{c}}$ in parallel to the $\mathrm{CPE}_{\mathrm{c}}$. This infers that, even in an early stage of the corrosion process, none of the coatings followed a classical barrier-type behaviour; instead, all of them were infiltrated by the $\mathrm{NaCl}$ solution all the way to the substrate to induce the electrode reactions (reactions (1)-(5)) even upon $1 \mathrm{~h}$ of immersion, probably due to their small thicknesses $(52 \pm 4 \mu \mathrm{m})$ that were unique in this work. Nevertheless, the Bode and Nyquist data of all the coatings upon $240 \mathrm{~h}$ and longer immersion times were found to be fitted more satisfactorily using a four-impedance model represented in Chart $1 \mathrm{~b}$. In addition to those in model $\mathrm{a}$, incorporated into model b is a fourth $R_{\text {diff }}-\mathrm{CPE}_{\mathrm{diff}}$ impedance element, which presumably suggests a significance of the finitelayer diffusion, ${ }^{37}$ in the later corrosion stage, that was caused by an occlusion of the coating channels for the corrosives infiltration by the corrosion products, basically $\mathrm{Fe}(\mathrm{OH})_{3}$ and $\mathrm{Fe}_{2} \mathrm{O}_{3}$ from reactions (4) and (5), respectively. According to models $\mathrm{a}$ and $\mathrm{b}$ for the respective immersion times of 1-24 $\mathrm{h}$ and 240960 h, added to Fig. 5 and 6 are all the simulated results (fitted curves), from which the evaluated $R_{\mathrm{c}}$, a measure of the anticorrosive performance of the coating itself without respect to its interfacing with the electrolyte solution as well as with the substrate, is plotted as a function of the immersion time for each composition of coating as shown in Fig. 7b (bar plots).

Qualitatively, for the EP44 coating specimen investigated (Fig. 6a), typical, single capacitive arcs of decreasing sizes were observed upon its respective 1 and $24 \mathrm{~h}$ immersion times in the $\mathrm{NaCl}$ solution, which usually were dictated by a dominant electrode process of reactions (1) and (2) in an initial stage of the corrosion. With an increase in the immersion time to $240 \mathrm{~h}$, the resulting smaller capacitive arc along with a successive short, sleek line probably was associated with the Warburg impedance due to a significance of dissolved oxygen diffusion at that time, ${ }^{38}$ which, characteristic of an additional electrode process caused by reactions (1) and (3), intensified the $\mathrm{Fe}^{3+}$ diffusion from accelerated tinplate corrosion across the substrate-coating interface. ${ }^{39}$ When the immersion time further was prolonged beyond $480 \mathrm{~h}$, it seemed that double capacitive arcs occurred upon each of the immersion times, whose combined size further reduced increasingly as the immersion time elapsed from 480 to $960 \mathrm{~h}$. These suggest that, while the immersion time of the EP44 coating specimen steadily was extended from 1 to $960 \mathrm{~h}$, its capacitive arc(s) size and thus anticorrosive performance were decreased monotonously, which arose obviously from progress in (i.e. intensification of) the corrosives diffusion across the coating that enhanced the electrode reactions causing the tinplate corrosion and hence increased the coating conductivity. ${ }^{30}$ For the TMDMS-grafted EP44 coating specimen (Fig. 6b), despite the observation that an upward tail (in a low frequency region) arose in addition to the capacitive arc (in the higher frequency region) upon its 1, 24 and $240 \mathrm{~h}$ of immersion, its anticorrosion behaviour generally 

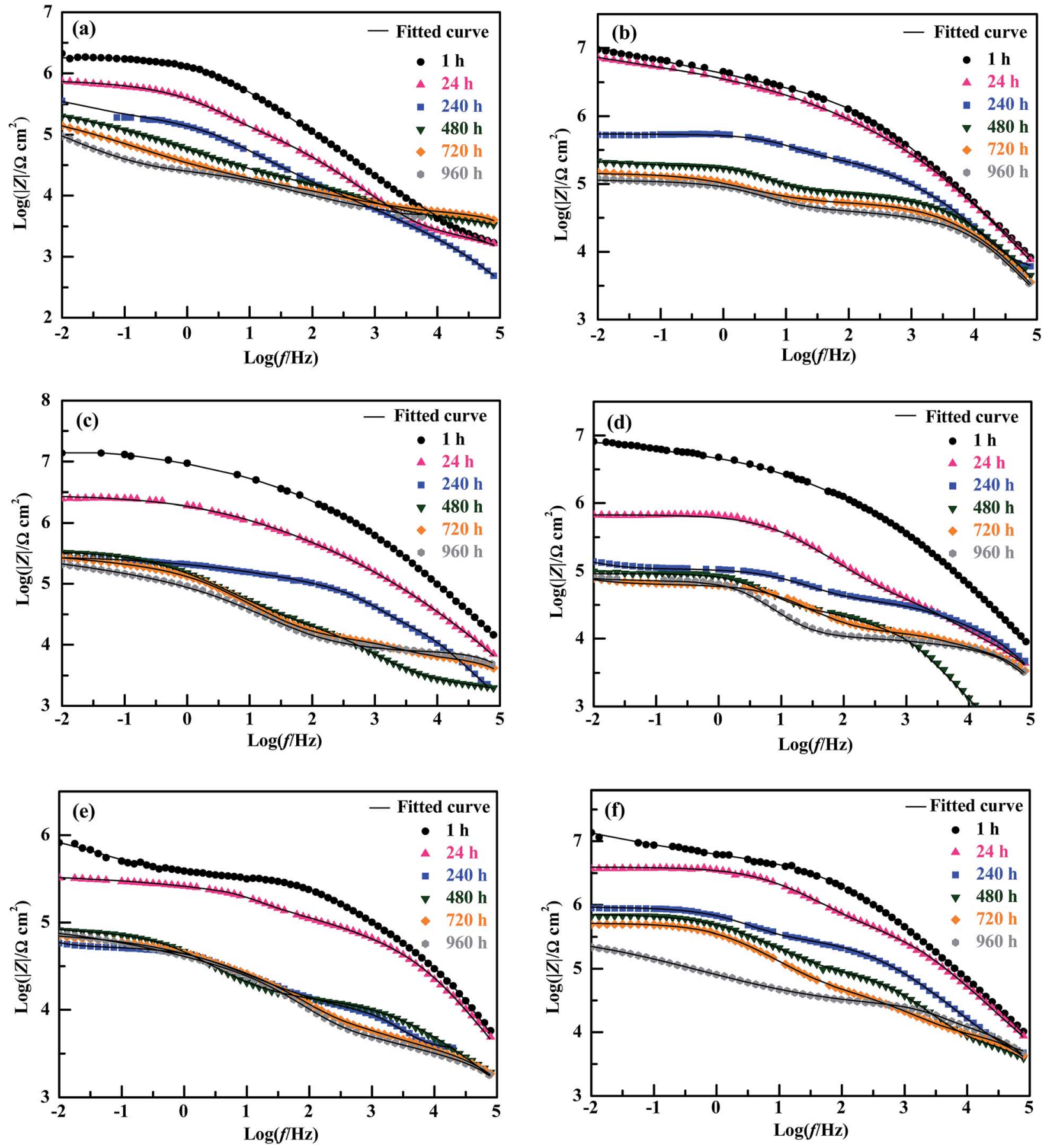

Fig. 5 Bode plots (i.e. plots of $\log |Z|$ vs. $\log f$ ) and their fitted curves by electrochemical equivalent circuit models for the corrosion inhibitive coatings, applied onto a 2 wt\% NaOH solution-treated tinplate substrate, of (a) a bisphenol-A based epoxy resin of an epoxy value of 0.440 (i.e. EP44), (b) a 3,3,3-trifluoropropylmethyldimethoxysilane (TMDMS) grafted EP44, and the mixtures of the TMDMS-grafted EP44 filled with different contents (wt\%): (c) 0.5; (d) 1; (e) 3; (f) 5 of 1,1,1,3,3,3-hexamethyldisilazane (HMDS) modified nanosilica powder that all are cured in an $n$-butanol/ xylene $(7 / 3 \mathrm{w} / \mathrm{w})$ mixed solution with an amino-terminated polyamide-based curing agent (i.e. G650), upon their different immersion times of 1 , $24,240,480,720$ and $960 \mathrm{~h}$.

resembled that of the EP44 coating specimen (Fig. 6a) in that (1) double capacitive arcs occurred on 480-960 h of immersion, some even with tails, and (2) the capacitive $\operatorname{arc}(\mathrm{s})$ size reduced steadily across the entire immersion process. However, upon any of all their immersion times (1-960 h), the capacitive arc(s) size of the TMDMS-grafted EP44 coating specimen (Fig. 6b) essentially was greater than that of the EP44 coating specimen (Fig. 6a) (note that the abscissa and ordinate scales of Fig. 6b both are considerably larger than those of Fig. 6a). This indicates qualitatively that the coating specimen of EP44 was improved in regard to anticorrosive performance upon its hydrophobic modification with the TMDMS grafts. 

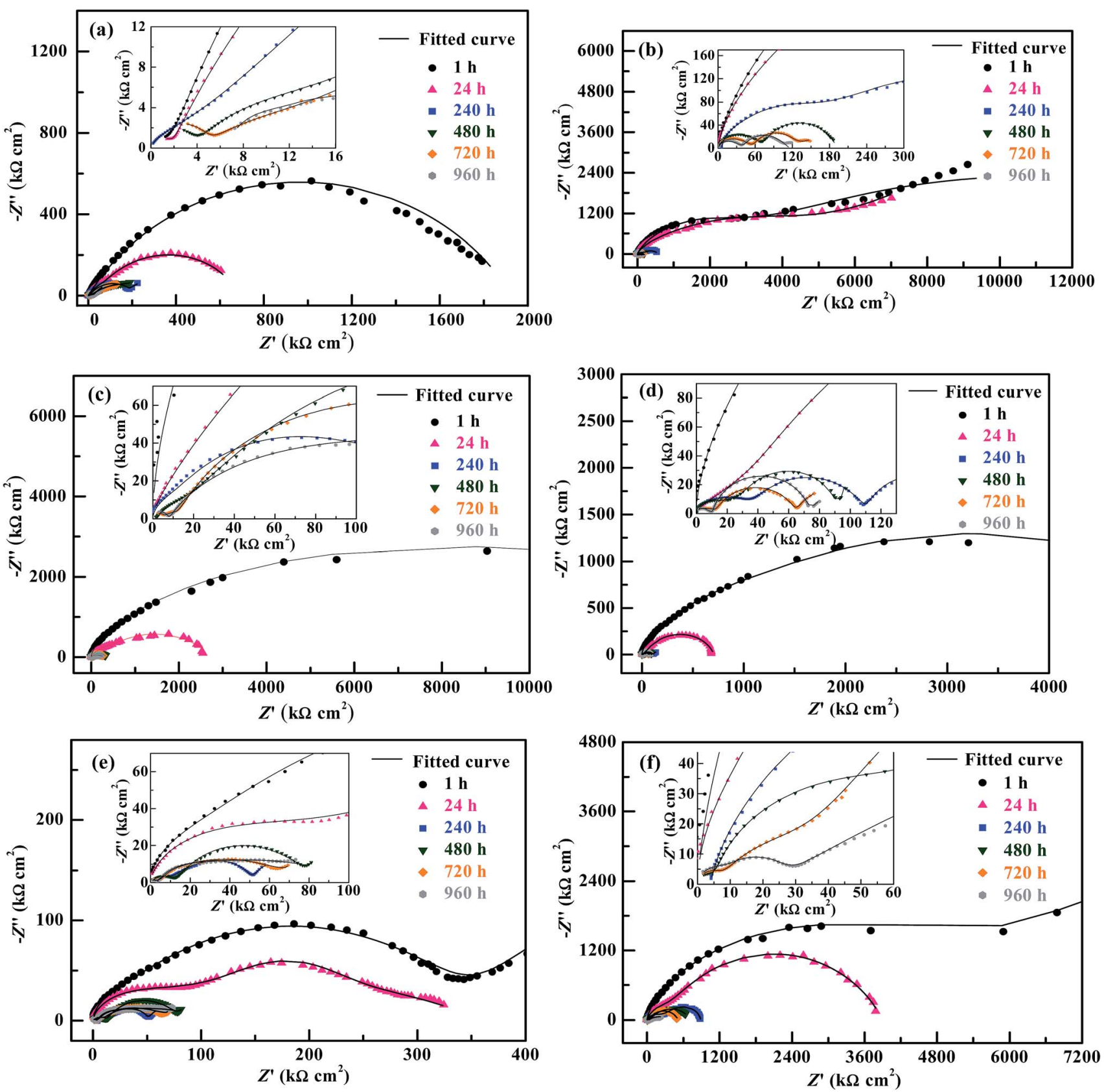

Fig. 6 Nyquist plots (i.e. plots of $-Z^{\prime \prime}$ vs. $Z^{\prime}$ ) and their fitted curves by electrochemical equivalent circuit models for the corrosion inhibitive coatings, applied onto a 2 wt\% NaOH solution-treated tinplate substrate, of (a) a bisphenol-A based epoxy resin of an epoxy value of 0.440 (i.e. EP44), (b) a 3,3,3trifluoropropylmethyldimethoxysilane (TMDMS) grafted EP44, and the mixtures of the TMDMS-grafted EP44 filled with different contents (wt\%): (c) 0.5; (d) 1; (e) 3; (f) 5 of 1,1,1,3,3,3-hexamethyldisilazane (HMDS) modified nanosilica powder that all are cured in an $n$-butanol/xylene (7/3 w/w) mixed solution with an amino-terminated polyamide-based curing agent (i.e. G650), upon their different immersion times of 1, 24, 240, 480, 720 and 960 h. In each of graphs a-f, the inset given is a magnification of the plots and fitted curves in a high frequency range (i.e. in the lower left corner).

More quantitatively, it was observed that both the $|Z|$ 's at $1 \times$ $10^{-2} \mathrm{~Hz}$ of the EP44 coating specimen (Fig. $\left.7 \mathrm{a}(1)\right)$ and of the TMDMS-grafted EP44 coating specimen (Fig. 7a(2)) decreased monotonically with their immersion time from 1 to $960 \mathrm{~h}$, which, agreeing with the qualitative results analysed from Fig. $6 \mathrm{a}$ and $\mathrm{b}$ respectively, corroborate that the anticorrosive performance of both the coating specimens was deteriorated steadily as their immersion proceeded. Nevertheless, with reference to the EP44 and TMDMS-grafted EP44 coatings themselves, although their anticorrosion behaviours (Fig. $7 \mathrm{~b}(1)$ and (2)) bore general resemblance to those of their respective specimens (Fig. 7a(1) and (2)) in that both the $R_{\mathrm{c}}$ 's essentially reduced with increasing the immersion time, the $R_{\mathrm{c}}$ of the TMDMS-grafted EP44 coating decreased little (Fig. $7 \mathrm{~b}(2)$ ) and, even, that of the EP44 coating turned to increase slightly (Fig. $7 \mathrm{~b}(1)$ ) both with increasing their immersion time beyond $480 \mathrm{~h}$. These presumably were ascribed to the significance, in a terminal stage of the corrosion process, of a barrier protection 
(a)

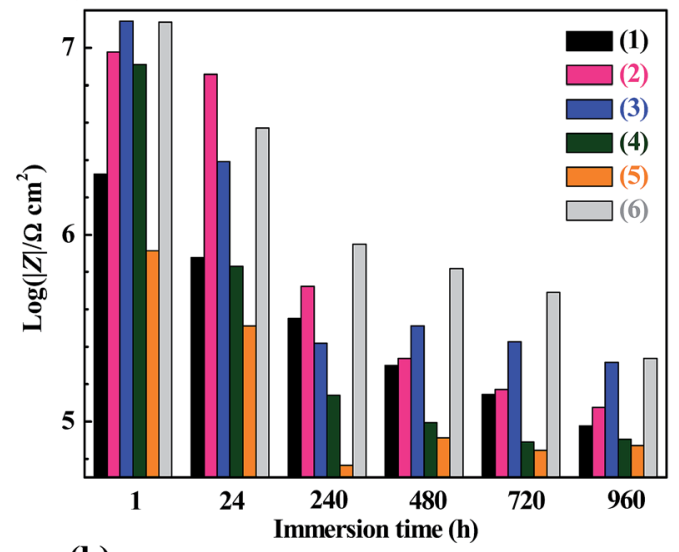

(b)

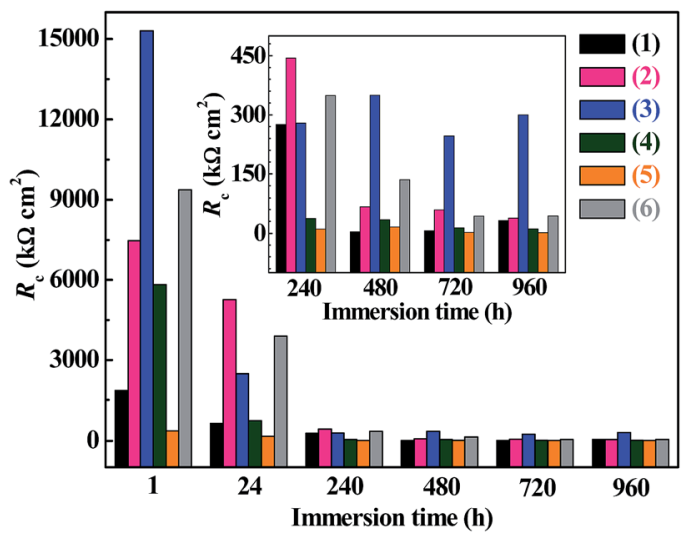

Fig. 7 Histograms of (a) the impedance magnitudes $(|Z|$ 's) at a frequency of $1 \times 10^{-2} \mathrm{~Hz}$ and (b) the coating resistances $\left(R_{\mathrm{c}}\right.$ 's) both as functions of the immersion time into an $\mathrm{NaCl}$ aqueous solution of $3.5 \mathrm{wt} \%$, for the corrosion preventive coatings, against a $2 \mathrm{wt} \% \mathrm{NaOH}$ solution-treated tinplate substrate, of (1) a bisphenol-A based epoxy resin of an epoxy value of 0.440 (i.e. EP44), (2) a 3,3,3-trifluoropropylmethyldimethoxysilane (TMDMS) grafted EP44, and the mixtures of the TMDMS-grafted EP44 filled with different contents (wt\%): (3) 0.5 ; (4) 1; (5) 3; (6) 5 of 1,1,1,3,3,3-hexamethyldisilazane (HMDS) modified nanosilica powder that all are cured in an $n$-butanol/ xylene $(7 / 3 \mathrm{w} / \mathrm{w})$ mixed solution with an amino-terminated polyamidebased curing agent (i.e. G650).

of the corrosion products deposited into the coating. ${ }^{18}$ More importantly, it is seen from Fig. 7 that increments of the resistive properties of the TMDMS-grafted EP44 coating occurred, when compared with those of the EP44 coating, in terms of both the $|Z|$ read typically at $1 \times 10^{-2} \mathrm{~Hz}\left(\right.$ Fig. $7 \mathrm{a}(1) v s$. (2)) and the $R_{\mathrm{c}}$ extrapolated by the models fits (Fig. $7 \mathrm{~b}(1)$ vs. (2)), at all the immersion times of 1, 24, 240, 480, 720 and $960 \mathrm{~h}$. These reveal quantitatively that the anticorrosive performance of the coating (i.e. both coating specimen and coating itself) of EP44 was enhanced upon its hydrophobic modification by the TMDMS grafting.

This anticorrosion superiority of the TMDMS-grafted EP44 coating could originate exclusively from the chemical structural changes of EP44 on the graft, which, as discussed in the last subsection, primarily gave rise to a significant improvement in its hydrophobicity (water $\theta$ from $83.8^{\circ}$ to $105.5^{\circ}$ ). It generally is (a)
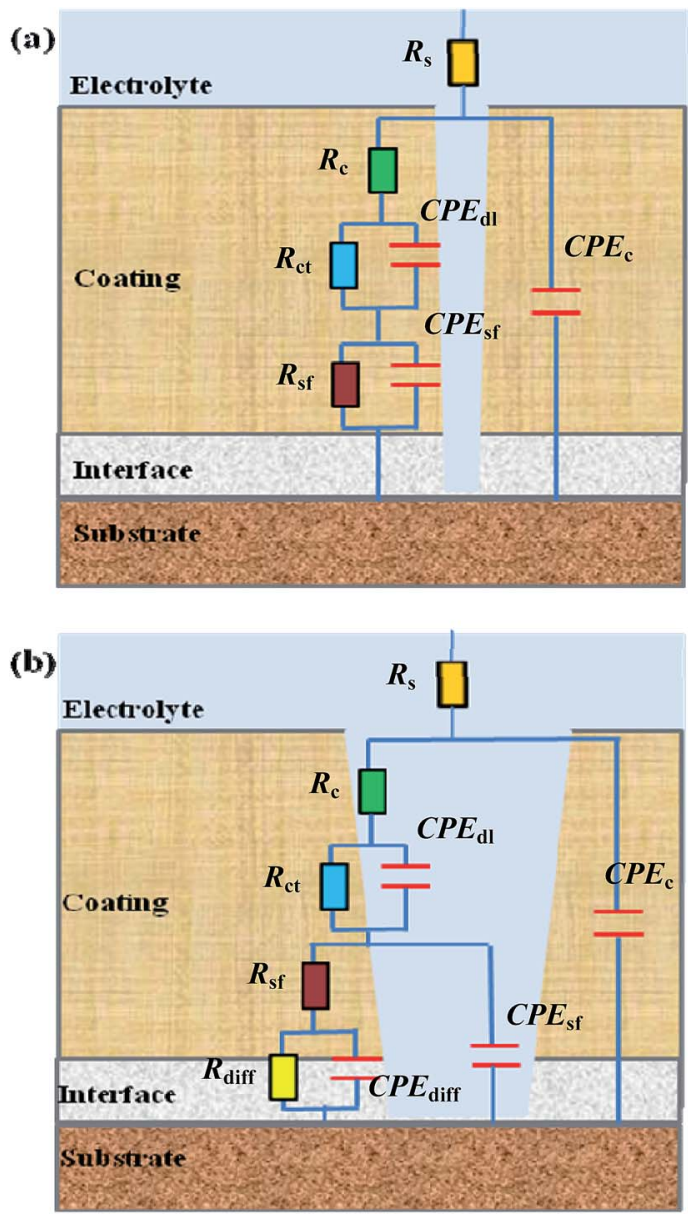

Chart 1 Two equivalent-circuit models used to fit the electrochemical impedance spectroscopy (EIS) experimental data presented in Fig. 5 and 6, where model a is appropriate for an early stage of the corrosion process (i.e. at short immersion times) while model $b$ is suitable for the later stage of the corrosion process (i.e. at longer immersion times) when the blockage effect of the corrosion product(s) is significant. In model a, the $R_{\mathrm{s}}, R_{\mathrm{c}}, R_{\mathrm{ct}}$ and $R_{\mathrm{sf}}$, respectively, represent the electrolyte solution resistance, coating resistance, charge-transfer resistances at the electrolyte-coating interface and from the coating to the substrate, while the $C P E_{c}, C P E_{d l}$ and $C P E_{s f}$, respectively, represent the coating capacitance, double-layer capacitances at the electrolyte-coating and coating-substrate interfaces; in model $\mathrm{b}$, the $R_{\text {diff }}$ and $C P E_{\text {diff, respectively, are the resistance and }}$ capacitance both associated with the finite-layer diffusion caused by an occlusion of the corrosives channels in the coating by the corrosion product(s), in addition to the presence of all the $R$ 's and CPE's shown in model a.

held that the protection of coatings against substrate corrosion may tentatively be classified into three mechanisms, barrier, sacrificial, and inhibitive, ${ }^{36}$ where the barrier capability to water, medium for corrosives, can further be defined by the adsorption ability of water onto coatings, the diffusion rate of water across coatings, and the accessibility of a substrate to water. ${ }^{40}$ In this work, the notably increased hydrophobicity (or water $\theta$ ) of the TMDMS-grafted EP44 coating indicates its much lower rates of water adsorption and diffusion that then also caused a well decreased water concentration adsorbed on the substrate, the three of which might effectively raise the $R_{\mathrm{ct}}, R_{\mathrm{c}}$ 
and $R_{\mathrm{sf}}$ of the Chart 1 models, respectively. Hence, it was this significantly stronger ability of hydrophobic barrier ${ }^{\mathbf{4 1}}$ that primarily was responsible for the improvement, stated above, in the anticorrosive performance of both the coating specimen and the coating itself of the TMDMS-grafted EP44. Besides, the unreacted, residual methoxy groups present in the TMDMS grafts, as evidenced by the NMR result (Fig. 2(c and f)) discussed in the first subsection, were subject to water absorption (i.e. digestion) via their hydrolysis, which decreased as well the water infiltration into the coating; this water-digestive "barrier", although minor, might also contribute to the anticorrosion enhancement.

An observation can be made from Fig. 6 and 7 as follows: qualitatively, the capacitive $\operatorname{arc}(\mathrm{s})$ size of the TMDMS-grafted EP44 coating specimen (Fig. 6b) was significantly greater than that of the EP44 coating specimen (Fig. 6a), and, more quantitatively, the $|Z|$ (Fig. $7 \mathrm{a}(2))$ and $R_{\mathrm{c}}($ Fig. $7 \mathrm{~b}(2))$ of the former were $0.5-1$ order of magnitude larger than those (Fig. $7 \mathrm{a}(1)$ and $\mathrm{b}(1)$ ) of the latter, both only upon their 1 and $24 \mathrm{~h}$ immersion times; on the longer times of immersion, all the increases in the capacitive $\operatorname{arc}(\mathrm{s})$ size, $|Z|$ and $R_{\mathrm{c}}$ were more or less minor from the graft modification. Nevertheless, since the immersion experiments of the two coating specimens virtually were simulated, expedited versions of their practical applications, this observation may not prevent us from judging the anticorrosive performance of the EP44 coating to significantly be improved upon its grafting with TMDMS, basically from the EIS data in the early stages of their experimental corrosion processes (i.e. on their 1 and $24 \mathrm{~h}$ immersion times).

\section{Effect of nanosilica content on the corrosion inhibition of composite coatings of a hydrophobic epoxy resin matrix}

For the composite coating specimens of the TMDMS-grafted EP44 filled with $0.5,1,3$ and $5 \mathrm{wt} \%$ of the HMDS-modified nanosilica, their respective Nyquist plot profiles (Fig. 6(c-f)) essentially were all similar to those of the EP44 (Fig. 6a) and TMDMS-grafted EP44 (Fig. 6b) coating specimens. These similarities include: (1) in an early stage of the corrosion (upon 1$480 \mathrm{~h}$ of immersion), a single capacitive arc occurred, sometimes along with an upward tail, while, in the later stage (on 24$960 \mathrm{~h}$ of immersion), double capacitive arcs arose, possibly followed by an upward tail as well, the critical immersion time for the early-to-later stage transition contingent apparently upon the corrosion inhibitive efficiency of the composite coating specimens (i.e. a stronger inhibition of corrosion dictated a longer critical immersion time); and (2) despite its possible fluctuations in the later stage, the capacitive arc(s) size generally reduced as the immersion (i.e. corrosion) progressed steadily. More quantitatively, for all the composite coatings investigated, both the $|Z|$ 's at $1 \times 10^{-2} \mathrm{~Hz}$ (Fig. 7a(3)-(6)) of the coating specimens and the $R_{\mathrm{c}}$ 's (Fig. $\left.7 \mathrm{~b}(3)-(6)\right)$ of the coatings themselves primarily were decreased with the immersion time until $960 \mathrm{~h}$, indicating that the corrosion inhibition (i.e. anticorrosive performance) of the coatings weakened on the whole as the corrosives infiltration into them intensified gradually.
However, abnormal variations (e.g. little decrease(s), slight increase(s), or their combination) in the $|Z|$ at $1 \times 10^{-2} \mathrm{~Hz}$ and/ or $R_{\mathrm{c}}$ occurred in the later stages of after 240,240, 240 and $720 \mathrm{~h}$ immersion times, respectively, for the composite coatings containing $0.5,1,3$ and $5 \mathrm{wt} \%$ of the modified nanosilica; again, these originated probably from the significance of a physical barrier, to the corrosives, provided by the corrosion products, basically $\mathrm{Fe}(\mathrm{OH})_{3}$ and $\mathrm{Fe}_{2} \mathrm{O}_{3}$, that were deposited into the composite coatings through cathodic reactions (4) and (5), respectively.

To discuss how the loading level ( $0-5 \mathrm{wt} \%)$ of the modified nanosilica affected the corrosion inhibition of the composite coatings, we now focus on comparisons, in Fig. 7, of their $|Z|$ (a) $\times 10^{-2} \mathrm{~Hz}$ or $R_{\mathrm{c}}$ values (including that of the TMDMSgrafted EP44 coating) upon each of all the immersion times. It is observed from Fig. 7a that, as the modified nanosilica content was raised from $0 \mathrm{wt} \%$ (Fig. $7 \mathrm{a}(2)$ ) steadily to $5 \mathrm{wt} \%$ (Fig. $7 \mathrm{a}(6)$ ), the $|Z|^{\text {'s }}$ at $1 \times 10^{-2} \mathrm{~Hz}$ upon $1,480,720$ and $960 \mathrm{~h}$ of immersion first increased, then decreased, and finally increased again, going through a maximum followed by a minimum at 0.5 and $3 \mathrm{wt} \%$ respectively, while those on 24 and $240 \mathrm{~h}$ of immersion first decreased and then increased, giving rise to a minimum still at $3 \mathrm{wt} \%$. These apparently contradictory observations (i.e. double extrema vs. a single extremum in the $|Z|)$ may actually be reconciled if one thinks that, specifically, the optimum nanosilica content for all the maxima in the $|Z|$ lay invariably between 0 and $0.5 \mathrm{wt} \%$. This means that all the $|Z|$ 's at $1 \times 10^{-2} \mathrm{~Hz}$ of the composite coating specimens upon their increasing immersion times (1-960 h) presumably followed the above double extrema rule, respectively, at critical 0-0.5 and $\sim 3 \mathrm{wt} \%$ contents of the modified nanosilica, as its concentration was enhanced from $0 \mathrm{wt} \%$ all the way to $5 \mathrm{wt} \%$. Interestingly enough, it is seen from Fig. $7 \mathrm{~b}(2)-(6)$ that the $R_{\mathrm{c}}$ of the composite coatings themselves seemed to exhibit exactly the same double extrema behaviour on each of their 1-960 h of immersion, rising to be maximised at a first critical content of $0-0.5 \mathrm{wt} \%$, then dropping to be minimised at a second, higher critical content of $\sim 3 \mathrm{wt} \%$, and finally rising again with steady increases in the nanosilica concentration from 0 to $5 \mathrm{wt} \%$. Since the reconciled $|Z| @ 1 \times 10^{-2} \mathrm{~Hz}$ and $R_{\mathrm{c}}$ were both measures of the corrosion inhibition of the composite coatings, the established double extrema (or dual critical concentrations) relationship obviously constituted the effect of the modified nanosilica content on the corrosion inhibition of the composite coatings having the hydrophobic TMDMS-grafted EP44 matrix, whose mechanism will be discussed in detail in the next subsection.

\section{A tentative dual critical concentrations mechanism of the nanosilica content effect}

As revealed from the water $\theta$ data of the composite coatings presented in Table 3, addition of the modified nanosilica by 0.5-5 wt\% changed little their hydrophobicity. This suggests that the found double-extrema behaviour in corrosion inhibition, respectively, at the dual critical nanosilica concentrations originated essentially from variations in the physical barrier 
ability of the nanosilica instead of in the hydrophobic barrier capability of the composite coatings. As mentioned in the Introduction, incorporating IC such as silica nanoparticles into an EPR coating has generally been shown to improve its corrosion inhibition by filling the defective pores (free volumes, voids, cracks, etc.) present that are susceptible to corrosives; ${ }^{24-28}$ however, too many IC nanoparticles beyond their critical concentration may deteriorate the corrosion inhibition due to an increase in the composite coating porosity from size exclusion of significantly aggregated IC particles. ${ }^{24,29}$ As such, only the maximum (i.e. a single extremum) in the corrosion inhibition has been reported of EPR/IC composite coatings at a critical concentration $\left(c_{\mathrm{c}}\right)$ of the IC over the $0-10 \mathrm{wt} \%$ IC loading levels investigated. Nevertheless in this work, we seemed to uniquely locate a second, higher $c_{\mathrm{c}}\left(c_{\mathrm{c} 2}\right)$ of the modified nanosilica, successively after its (first) $c_{\mathrm{c}}\left(c_{\mathrm{c} 1}\right)$ causing a maximum in the corrosion inhibition, that gave rise to a minimum in the corrosion inhibition of the composite coatings of the hydrophobic TMDMS-grafted EP44 matrix.

This nanosilica content effect, mechanistically referred to as a dual critical concentrations behaviour, is therefore interpreted tentatively with reference to Scheme 5. First, be advised that a rise in the modified nanosilica concentration, due to its increased tendency to aggregate especially thermally during the vacuum drying at $80{ }^{\circ} \mathrm{C}$ for $\geq 8 \mathrm{~h}$ ( $c f$. Scheme 3 ), led to an increase in its (mean) size. When a small concentration of them were added to the TMDMS-grafted EP44 coating full of increasing sizes of defective pores that were accessible to the corrosives, the nanosilica particles, at their small size, partially filled the pores whose sizes were larger, mostly at their plural form (Scheme 5a); this filling acted as a degree of physical barrier to the infiltration of the corrosives into the pores, reducing the effective cathodic area (i.e. pore volume) for the electrode reactions and hence improving the corrosion inhibition of the coating. As their concentration was raised to the $c_{\mathrm{c} 1}$, the silica nanoparticles of an increased size filled, to the most extent, all the pores of larger sizes present (Scheme 5b), maximising the physical barrier protection and thus the corrosion inhibition of the coating. However, while their concentration further was increased above the $c_{\mathrm{c} 1}$, the silica nanoparticles were large in size enough to be size-excluded from (i.e. vacate) such many pores of smaller sizes that the ability of physical barrier to the corrosives began to weaken, which deteriorated the corrosion inhibition of the coating; concomitant with this was a gradual transition of the particles filling mode from plural to singular (Scheme 5c). Typically, the moment their concentration was enhanced up to the $c_{\mathrm{c} 2}$, the nanosilica particles grew too large in size to fill any of all the increasing sizes of pores due to their entire size exclusion (Scheme $5 \mathrm{~d}$ ), which maximised the effective cathodic area and thereby minimised the corrosion inhibition of the coating. And, ultimately, once their concentration was increased beyond the $c_{\mathrm{c} 2}$, the silica particles, all sizeexcluded from the pores, became increasingly larger in size and denser in number, thus subject to formation of at least locally continuous, outside-of-pore physical barriers (Scheme 5e); this behaviour, cutting off to an extent the percolated diffusion of

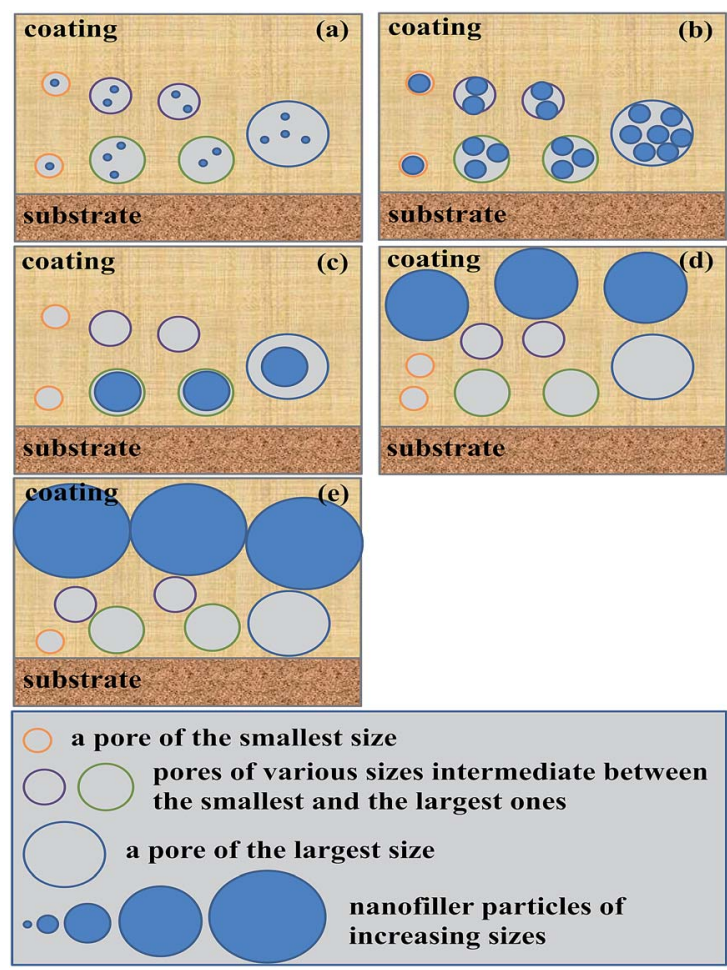

Scheme 5 Schematic of a tentative, dual critical concentrations mechanism for the interpretation of the effect of nanofiller content on the corrosion inhibition, by filling the defective pores (free volumes, voids, cracks, etc.) for the diffusion detour of corrosives, of hydrophobic polymer-matrix composite coatings, where, with a steady enhancement of its concentration from (a) to (d), the nanofiller becomes increasingly larger in (mean) size due to its aggregation: (a) at their small concentrations and sizes, the nanofiller particles fill partially and largely plurally the pores of different sizes present in the coatings; (b) as their concentration is increased to a critical value, $c_{c 1}$, the nanoparticles fully occupy (i.e. jam) all the different-sized pores, mostly at their plural form; (c) when their concentration further is enhanced beyond the $c_{c 1}$, the nanoparticles are large enough to increasingly be size-excluded from part of the pores of smaller sizes; (d) while their concentration is up to a second, higher critical value, $c_{\mathrm{c} 2}$, the nanofiller particles are so large (i.e. larger than the largest size pores) as to fill none of all the sizes of pores; and (e) as their concentration ultimately is raised beyond the $c_{\mathrm{c} 2}$, the aggregated particles become extremely larger and denser, acting as an increasingly solid, outside-of-pore barrier to the diffusion of corrosives.

the corrosives, obviously turned to enhance the corrosion inhibition of the coating again.

As analysed in the last subsection, the $c_{\mathrm{c} 1}$ and $c_{\mathrm{c} 2}$, a couple of indices of the dual critical concentrations mechanism, were quantified as $0-0.5$ and $\sim 3 \mathrm{wt} \%$, respectively, for the composite coatings of the TMDMS-grafted EP44 filled with the HMDSmodified nanosilica. This is in sharp contrast to a much higher $c_{\mathrm{c}}$ (i.e. $\left.c_{\mathrm{c} 1}\right), \sim 4 \mathrm{wt} \%$, of the single critical concentration mechanism reported for the composite coatings of a bisphenolA based EPR (Epikote 828) filled with a GPTMS-treated nanosilica. ${ }^{30}$ The rationale behind this contrast may lie in the following. Most importantly, owing to the significantly more hydrophobic nature of the TMDMS-grafted EP44 matrix than the Epikote 828 matrix, there were much fewer defective pores 
(or channels made of them), present in the composite coatings investigated in this work, that were accessible to water and the corrosive solutes (molecules, ions, etc.) therein. Secondly, by reducing the nanosilica hydroxyls more upon their higherconversion reaction with HMDS than with GPTMS, the finer silica nanoparticles from more decreased aggregation prepared in this work filled more effectively the coating pores especially those small-sized ones. Thirdly, compared with the direct (single-step) cure reported of Epikote 828 at $120{ }^{\circ} \mathrm{C}$ for $30 \mathrm{~min}$, the much-milder, step cure of the TMDMS-grafted EP44 ( $c f$. step 6 to 7 of Scheme 3: cure in fume hood at RT for 7 days, followed by postcure in vacuo by a ramp to $70{ }^{\circ} \mathrm{C}$ in $1 \mathrm{~h}$ and then a hold there for $3 \mathrm{~h}$ ) caused considerably fewer and smaller defective pores, from greatly slowed solvent evaporation, that intrinsically were developed in the composite coatings. These three factors contributed, sequentially decreasingly while all significantly, to the sharp decrease in the $c_{\mathrm{c} 1}, e . g$. from $\sim 4 \mathrm{wt} \%$, to only $0-0.5 \mathrm{wt} \%$, which, further, interestingly was followed by the occurrence of the higher $c_{\mathrm{c} 2}$ at $\sim 3 \mathrm{wt} \%$ that would not have otherwise arisen over the 0-10 wt $\%$ nanofiller contents investigated.

\section{Conclusions}

TMDMS has successfully been grafted onto EP44 molecules to substitute trifluoropropyls for hydroxyls via significant transesterification (i.e. alcoholysis) of the TMDMS methoxyls with the EP44 hydroxyls, as corroborated by FTIR and ${ }^{1} \mathrm{H}$ NMR spectroscopies, under anhydrous DBTDL-catalyst conditions at $95^{\circ} \mathrm{C}$ for $8 \mathrm{~h}$. Meanwhile, a nanosilica powder of $30 \mathrm{~nm}$ in mean diameter has successfully been modified with HMDS to convert its surface hydroxyls into methyls through electrophilic substitution of the nanosilica hydroxyls by the HMDS trimethylsilyls at $50{ }^{\circ} \mathrm{C}$ for $20 \mathrm{~h}$, as confirmed by FTIR spectroscopy and TGA. For comparative investigations, coatings of uniform thicknesses $(52 \pm 4 \mu \mathrm{m})$, onto an $\mathrm{NaOH}$ solution-treated tinplate substrate, of EP44, of the TMDMS-grafted EP44, and of the composites of the TMDMS-grafted EP44 filled with increasing contents $(0.5,1$, 3 and $5 \mathrm{wt} \%$ ) of the HMDS-modified nanosilica have been prepared from an $n$-butanol/xylene solution by the same mild, step cure with an amino-terminated polyamide-based curative. It is revealed from contact angle $(\theta)$ measurements of the six coatings that the $\theta$ of water against, and thus the hydrophobicity of, the EP44 coating considerably is increased from $83.8^{\circ}$ to $105.5^{\circ}$ upon its grafting with TMDMS, which then changes little with further addition of the increasing concentrations of the modified nanosilica. Likely, this is due predominantly to the introduction of superhydrophobic trifluoropropyl groups, as well as secondarily to a reduction in the hydrophilic hydroxyl concentration present, both upon the graft modification.

The anticorrosion processes of the six coating specimens, upon immersion into an electrolyte (i.e. $\mathrm{NaCl}$ ) aqueous solution, have therefore been characterised by EIS Bode plots, i.e. plots of the logarithm of impedance magnitude $(\log |Z|) v s$. that of frequency $(\log f)$, and Nyquist plots, i.e. plots of the opposite of capacitive reactance $\left(-Z^{\prime \prime}\right)$ vs. resistance $\left(Z^{\prime}\right)$. These experimental data have further been simulated satisfactorily by appropriate electrochemical equivalent-circuit models, from which the coating resistance $\left(R_{\mathrm{c}}\right)$, a measure of the anticorrosive performance of the coating itself without respect to its interfacing with the electrolyte solution as well as with the substrate, has been evaluated for each of the coatings upon each of the immersion times $(1,24,240,480,720$ and $960 \mathrm{~h})$ investigated. Generally, the capacitive arc(s) sizes observed from the Nyquist plots, the $|Z|$ 's at $1 \times 10^{-2} \mathrm{~Hz}$ read from the Bode plots, and/or the $R_{\mathrm{c}}$ 's extrapolated from the model fits are decreased with increasing the immersion time until $240-720 \mathrm{~h}$, while then vary abnormally (e.g. decrease little, increase slightly, or both (i.e. fluctuate)) as the immersion time is increased further, for all the six coatings under investigation. These indicate that the anticorrosive performance of the coatings deteriorates steadily in an early stage of the corrosion process due to progress in (i.e. intensification of) the corrosives (i.e. water, electrolyte ( $\mathrm{NaCl}$ ), oxygen, other molecules and ions) infiltration into their pores, whereas changes little in the later stage presumably owing to a significance of the cathodic deposition of the corrosion products (basically $\mathrm{Fe}(\mathrm{OH})_{3}$ and $\mathrm{Fe}_{2} \mathrm{O}_{3}$ ) into the coatings, which competes against the corrosive diffusion by occluding the coating pores or channels to act as a physical barrier to the corrosives.

More importantly, it has been found that the capacitive arc(s) size, the $|Z| @ 1 \times 10^{-2} \mathrm{~Hz}$, and the $R_{\mathrm{c}}$ of the TMDMS-grafted EP44 coating, respectively, all are larger than those of the EP44 coating upon each of the immersion times, revealing that the anticorrosive performance of the former evidently is improved compared with the latter, as a consequence probably of the significantly increased hydrophobic barrier to the corrosives mediated by water, as well as possibly of the water digestion (i.e. "chemical barrier") from hydrolysis of the residual, unreacted TMDMS methoxyls, both present in the coating upon the graft modification. Further, when the TMDMS-grafted EP44 coating is filled with the increasing contents $(0-5 \mathrm{wt} \%)$ of the modified nanosilica, the little changes in its hydrophobicity dictate that the anticorrosive performance of the composite coatings has to be dominated by a corrosion inhibition mechanism by the nanosilica particles filling the defective pores (free volumes, voids, cracks, etc.), present in the coatings, that are accessible to the corrosives. In other words, the silica nanoparticles may act as physical barriers in the pores to the diffusion of water, the corrosive molecules and ions therein, making them take a significant detour to the substrate. Still from the associated, reconciled EIS data (capacitive $\operatorname{arc}(\mathrm{s})$ sizes and $|Z|$ 's at $\left.1 \times 10^{-2} \mathrm{~Hz}\right)$ and simulations $\left(R_{\mathrm{c}} \mathrm{s}\right)$, it has interestingly been found that, as the nanosilica particles concentration steadily is raised from 0 to $5 \mathrm{wt} \%$, the corrosion inhibition of the composite coatings first intensifies probably thanks to an enhancement of the filling rate of the pores, subsequently weakens presumably due to an increase in the porosity from increased size-exclusion of aggregated nanoparticles from the pores, and finally improves again possibly owing to a densification of the outside-of-pore barriers of greatly aggregated, size excluded particles to the corrosives. This constitutes dual critical concentrations of the nanosilica at $0-0.5$ and $\sim 3 \mathrm{wt} \%$, respectively, that give rise to two extrema (a maximum followed 
by a minimum) in the corrosion inhibition of the composite coatings.

Particularly worth noting is that the dual critical concentrations behaviour discovered above contrasts with a single critical concentration of a GPTMS-modified nanosilica at $\sim 4 \mathrm{wt} \%$, much higher than the $0-0.5 \mathrm{wt} \%$, that causes the maximum in the corrosion inhibition of composite coatings of Epikote 828 matrix. ${ }^{30}$ Presumably, the rationale behind this contrast lies primarily in the much more hydrophobic nature of the TMDMSgrafted EP44 matrix that leads to fewer coating pores or channels accessible to the water-mediated corrosives, secondarily in the higher pore-filling efficiency of the less aggregated, finer HMDS-modified silica nanoparticles from their more reduced surface hydroxyls, and thirdly in the fewer and smaller total pores or channels developed within the coatings studied in this work from their milder, step cure process, all of which contribute to the minimisation of the (first) critical concentration to $0-0.5 \mathrm{wt} \%$ and hence the occurrence of the second, higher critical concentration at $\sim 3 \mathrm{wt} \%$ across the $0-10 \mathrm{wt} \%$ nanosilica contents investigated. To our knowledge, this dual critical concentrations behaviour has been the first work reported in the literature to rule the corrosion inhibition-nanosilica content relationship of composite coatings of an EPR matrix, which may universally be extended to other composite coatings of an organic polymer matrix filled with different contents of an IC nanofiller for corrosion prevention of a metallic substrate.

\section{Conflicts of interest}

There are no conflicts of interest to declare.

\section{Acknowledgements}

We acknowledge with gratitude that this work was financially supported by the Natural Science Foundation of the Hubei Province of China (Contract no. 2016CFB205 and 2014CFA094), as well as by the National Natural Science Foundation of China (Contract no. 51601133) and the Select Overseas Chinese Scholars Science and Technology Activities Foundation of the Ministry of Human Resources and Social Security of China (Contract no. [2013]277).

\section{References}

1 X. Lu, Y. Liu, C. L. Zhou, W. F. Zhang and Z. Xin, RSC Adv., 2016, 6, 5805.

2 T. H. Tran, A. Vimalanandan, G. Genchev, J. Fickert, K. Landfester, D. Crespy and M. Rohwerder, Adv. Mater., 2015, 27, 3825.

3 M. Behzadnasab, S. M. Mirabedini, K. Kabiri and S. Jamali, Corros. Sci., 2011, 53, 89.

4 Z. Li, B. Y. Qin, X. Y. Zhang, K. Wang, Y. Wei and Y. Ji, RSC Adv., 2015, 5, 104451.

5 L. B. Feng, Y. H. Che, Y. H. Liu, X. H. Qiang and Y. P. Wang, Appl. Surf. Sci., 2013, 283, 367.
6 F. Luo, Q. Li, X. K. Zhong, H. Gao, Y. Dai and F. N. Chen, Mater. Corros., 2012, 63, 148.

7 M. Qian, A. M. Soutar, X. H. Tan, X. T. Zeng and S. L. Wijesinghe, Thin Solid Films, 2009, 517, 5237.

8 B. Ramezanzadeh, E. Raeisi and M. Mahdavian, Int. J. Adhes. Adhes., 2015, 63, 166.

9 R. Suleiman, H. Dafalla and B. E. Ali, RSC Adv., 2015, 5, 39155.

10 S. V. Harb, F. C. dos Santos, B. L. Caetano, S. H. Pulcinelli, C. V. Santilli and P. Hammer, RSC Adv., 2015, 5, 15414.

11 A. M. Beccaria, G. Padeletti, G. Montesperelli and L. Chiaruttini, Surf. Coat. Technol., 1999, 111, 240.

12 T. O. Siyanbola, K. Sasidhar, B. Anjaneyulu, K. P. Kumar, B. V. S. K. Rao, R. Narayan, O. Olaofe, E. T. Akintayo and K. V. S. N. Raju, J. Mater. Sci., 2013, 48, 8215.

13 A. M. Mikhailova, M. Tamboura and M. Q. Jia, Silicon, 2012, 4, 197.

14 W. G. Ji, J. M. Hu, J. Q. Zhang and C. N. Cao, Corros. Sci., 2006, 48, 3731.

15 X. Yuan, Z. F. Yue, X. Chen, S. F. Wen, L. Li and T. Feng, Prog. Org. Coat., 2015, 86, 41.

16 Z. Y. Wang, F. C. Liu, E. H. Han and W. Ke, Mater. Corros., 2013, 64, 54.

17 L. Mathivanan and S. Radhakrishna, Anti-Corros. Methods Mater., 1998, 45, 301.

18 X. Y. Lu, Y. Zuo, X. H. Zhao and Y. M. Tang, Corros. Sci., 2012, $60,165$.

19 S. H. Zaferani, D. Zaarei, I. Danaee and N. Mehrabian, J. Adhes. Sci. Technol., 2014, 28, 151.

20 S. A. Kumar, T. Balakrishnan, M. Alagar and Z. Denchev, Prog. Org. Coat., 2006, 55, 207.

21 M.-Y. Jiang, L.-K. Wu, J.-M. Hu and J.-Q. Zhang, Corros. Sci., 2015, 92, 127.

22 P. Gupta and M. Bajpai, Adv. Chem. Eng. Sci., 2011, 1, 133.

23 W. G. Ji, J. M. Hu, L. Liu, J. Q. Zhang and C. N. Cao, Surf. Coat. Technol., 2007, 201, 4789.

24 F. Ansari, R. Naderi and C. Dehghanian, RSC Adv., 2015, 5, 706.

25 C. Motte, M. Poelman, A. Roobroeck, M. Fedel, F. Deflorian and M.-G. Olivier, Prog. Org. Coat., 2012, 74, 326.

26 X. M. Shi, T. A. Nguyen, Z. Y. Suo, Y. J. Liu and R. Avci, Surf. Coat. Technol., 2009, 204, 237.

27 L. Allie, J. Thorn and H. Aglan, Corros. Sci., 2008, 50, 2189.

28 M. C. Lai, K. C. Chang, J. M. Yeh, S. J. Liou, M. F. Hsieh and H. S. Chang, Eur. Polym. J., 2007, 43, 4219.

29 V. Palanivel, D. Q. Zhu and W. J. van Ooij, Prog. Org. Coat., 2003, 47, 384 .

30 A. Ghanbari and M. M. Attar, J. Ind. Eng. Chem., 2015, 23, 145.

31 Y. F. Li and J. Cheng, Adv. Mater. Res., 2006, 11, 379.

32 V. R. Shayapov, M. N. Chagin and Y. M. Rumyantsev, Inorg. Mater., 2016, 52, 630.

33 E. Vyhmeister, H. V. González Lorenzo, R. Bozo, R. R. Maecker, A. Muscat, L. A. Estévez and D. Suleiman, Chem. Eng. Commun., 2016, 203, 908.

34 B. Xie and A. J. Muscat, Microelectron. Eng., 2004, 76, 52.

35 J. He, X. L. Li, D. Su, H. M. Ji, X. Zhang and W. S. Zhang, J. Mater. Chem. A, 2016, 4, 5632. 
36 M. J. Palimi, M. Rostami, M. Mahdavian and B. Ramezanzadeh, J. Coat. Technol. Res., 2015, 12, 277.

37 X. Y. Lu, Y. Zuo, X. H. Zhao, Y. M. Tang and X. G. Feng, Corros. Sci., 2011, 53, 153.

38 Y. J. Qiang, S. T. Zhang, L. Guo, X. W. Zheng, B. Xiang and S. J. Chen, Corros. Sci., 2017, 119, 68.
39 X. Yuan, Z. F. Yue, X. Chen, S. F. Wen and L. Li, Prog. Org. Coat., 2015, 78, 1.

40 X. Yuan, Z. F. Yue, X. Chen, S. F. Wen, L. Li and T. Feng, J. Coat. Technol. Res., 2015, 12, 1.

41 R. C. Zeng, L. J. Liu, T. T. Pang, F. Zhang, W. W. Zhang, S. Q. Li, H. Z. Cui and E. H. Han, Acta Metall. Sin., 2015, 28, 373. 